Atmos. Chem. Phys., 13, 8315-8333, 2013

www.atmos-chem-phys.net/13/8315/2013/

doi:10.5194/acp-13-8315-2013

(c) Author(s) 2013. CC Attribution 3.0 License.

\title{
Pauci ex tanto numero: reduce redundancy in multi-model ensembles
}

\author{
E. Solazzo ${ }^{1}$, A. Riccio ${ }^{2}$, I. Kioutsioukis ${ }^{1,3}$, and S. Galmarini ${ }^{1}$ \\ ${ }^{1}$ European Commission, Joint Research Centre, Institute for Environment and Sustainability, Ispra, Italy \\ ${ }^{2}$ Department of Applied Science, University of Naples "Parthenope", Napoli, Italy \\ ${ }^{3}$ Region of Central Macedonia, Thessaloniki, Greece
}

Correspondence to: S. Galmarini (stefano.galmarini@jrc.ec.europa.eu)

Received: 9 January 2013 - Published in Atmos. Chem. Phys. Discuss.: 21 February 2013

Revised: 9 July 2013 - Accepted: 10 July 2013 - Published: 22 August 2013

\begin{abstract}
We explicitly address the fundamental issue of member diversity in multi-model ensembles. To date, no attempts in this direction have been documented within the air quality (AQ) community despite the extensive use of ensembles in this field. Common biases and redundancy are the two issues directly deriving from lack of independence, undermining the significance of a multi-model ensemble, and are the subject of this study. Shared, dependant biases among models do not cancel out but will instead determine a biased ensemble. Redundancy derives from having too large a portion of common variance among the members of the ensemble, producing overconfidence in the predictions and underestimation of the uncertainty. The two issues of common biases and redundancy are analysed in detail using the AQMEII ensemble of AQ model results for four air pollutants in two European regions. We show that models share large portions of bias and variance, extending well beyond those induced by common inputs. We make use of several techniques to further show that subsets of models can explain the same amount of variance as the full ensemble with the advantage of being poorly correlated. Selecting the members for generating skilful, non-redundant ensembles from such subsets proved, however, non-trivial. We propose and discuss various methods of member selection and rate the ensemble performance they produce. In most cases, the full ensemble is outscored by the reduced ones. We conclude that, although independence of outputs may not always guarantee enhancement of scores (but this depends upon the skill being investigated), we discourage selecting the members of the ensemble simply on the basis of scores; that is, independence and skills need to be considered disjointly.
\end{abstract}

\section{Introduction}

Geophysical modelling nowadays relies, among other techniques, on ensemble methods to improve predictive skills, assess performance and quantify uncertainties. This is also the case for atmospheric sciences, where climate and air quality (AQ) models are often treated as ensembles of an arbitrary collection of models results belonging to the same family, sharing similar structure and resolution ("ensembles of opportunity", as defined e.g. by Tebaldi and Knutti, 2007). Just as human beings normally consult a number of sources prior to making a decision (see for example the "trillion dollar garden party" analogy adopted by Knutti, 2010), the advantage of treating the information from several sources into ensembles relies on the fundamental assumption that information coming from multiple sources allows for an estimation of the quality of the former, in line with the "Principle of multiple explanations" proposed by the Greek philosopher Epicurus (341-270 BC), which says that for an optimal solution of a concrete problem, we have to take into consideration all the hypotheses that are consistent with the input data. One of the main strengths of multiple estimations derives from the independence of the sources. In fact, if the information came from similar or dependant sources, the net result would be a biased and overconfident estimate. In our view, in multi-model (MM) ensemble practices, the issue of member independence has been often overlooked and taken for granted. However, due to the fact that members of an ensemble are phenotypically similar (Potempski and Galmarini, 2009), caution is required. To avoid ambiguous interpretations we define: 
- Independence, a formal property, when the joint probability distribution function (PDF) of two or more models is derived from the product of single PDFs (Cover and Thomas, 2006). This is the rigorous definition of independence, though the joint PDF is difficult to estimate in practice.

- Uncorrelation, referring to the situation where model's outputs are linearly independent. This is the mostapplied proxy to independence. The outputs of independent models are uncorrelated, but uncorrelation does not guarantee independence.

- Diversity, a qualitative property. Models are said to be diverse when they are developed starting from different conceptual basis and are based on different causal assumptions. Their outputs (and errors) can be correlated. Proving diversity has the same practical difficulties to proving independence (in general models are the numerical coding of fundamental physical processes, and it is likely all models have at least this in common). Similarity is the opposite of diversity, and can be defined when models are developed from the same conceptual basis or share a number of elements that make them similar. Outputs and errors of similar models are expected to be highly correlated.

- Redundancy, when two or more models, dependent or not, have correlated outputs. It is more informative than correlation as redundancy is related to the amount of explained variance (Legendre and Legendre, 1998). In the case of mutual correlations of model pairs, the redundancy reduces to the coefficient of determination, $R^{2}$, the square of the correlation coefficient. Redundancy is the primary effect of model similarity, and applies to both model outputs and their errors.

The lack of independence of members in ensemble treatment is not at all new. Despite the empirical evidence of the superior performance of average of models in some cases (Fiore et al., 2009; van Loon et al., 2007; Vautard et al., 2009; Pierce et al., 2009; Galmarini et al., 2004; Potempski et al., 2008), it is known that models share similar deficiencies. Several studies have demonstrated that similarities of model errors are statistically significant beyond doubt, thus questioning the effectiveness of "blindly" combining models into ensembles. Nonetheless, the problem of member (and error) similarities has received little attention by the climate modelling community, as recently recognised by Pirtle et al. (2010), and even less by the AQ community, where the theoretical work by Potempski and Galmarini (2009) and the attempts by Riccio et al. (2012) and Solazzo et al. (2012a) remain the only studies, to the best of our knowledge, dedicated to the issue.

Independence of models can be sought in the form of different structures (proportion of parameterisations shared by the models), or, from an information science point of view, as the possibility to express the combined error PDF in terms of product of single PDFs (Abramowitz, 2010; Potempski and Galmarini, 2009). Ideally, perturbation of model parameters and associated uncertainty on model output could serve this scope, as suggested by Tebaldi and Knutti (2007), but this is often impractical. The strategy common to the (few) studies that directly investigate model diversity consists in attributing model independence only from the analysis of the output they produce. In particular, Potempski and Galmarini (2009) showed that, by relaxing the condition of model independence to that of model associativity, a robust theoretical framework could be built from which precise mathematical formulations could be drawn. Associativity is measured by the covariance or by the correlation of pairs of model outputs. However, caution is needed as "it is possible that two models could agree with respect to outputs despite being based on different casual assumption" (Pirtle et al., 2010). Thus, when looking at the correlation of model outputs as a metric for defining independence, diverse models producing correlated outputs would be erroneously considered as dependant. Uncorrelation of the outputs is a necessary but insufficient condition to guarantee independence. Furthermore, the similarity of the results from two diverse models is valuable information for assessing model accuracy and uncertainty.

Models are intrinsically wrong due to their numerical nature, imprecise input data and limited understanding of the atmospheric chemical-physical processes. What is important is that models have independent systematic errors for the biases to cancel out when models are combined into ensembles. In the impossibility of an a priori assessment of the independence of ensemble members, model bias is an excellent parameter to investigate the ensemble member interdependence. Shared biases determine the direction of the agreement of models, making it therefore essential to select models whose errors are independent and can average out. Moreover, a MM ensemble for which all biases have the same sign and value may give the false impression of accuracy, which is often confused with precision: the agreement of models to precisely predict the same (biased) result is confused with accuracy of models, which implies homogeneously distributed biases around measurements (Potempski and Galmarini, 2009).

To date, the link between ensemble accuracy and diversity of members is not well defined. As noted by Abramowitz et al. (2010) the accuracy of an ensemble of diverse members is not in theory guaranteed to be higher than a redundant ensemble, in light of the "error decomposition dilemma", a problem which has gathered massive attention in particular in the information technology community for classification problems (e.g. Brown et al., 2005). The mean square error (MSE) (as a metric for accuracy) of an ensemble of models can be decomposed into the sum of the variance (var), the covariance (cov) and the bias: 


\section{Scopes, data and method}

$\mathrm{MSE}=\frac{\mathrm{var}}{M}+\left(1-\frac{1}{M}\right) \operatorname{cov}+(\mathrm{bias})^{2}+\operatorname{var}(\mathrm{obs})$,

where obs are the observations and $M$ is the size of the ensemble (for semplicity in Eq. (1) we assume the same variance for all members). The error of the ensemble is sum of two positive terms (variance and bias squared), and there is no way to simply minimise the bias without enhancing the variance: accuracy cannot be gained at the expense of precision. With the covariance being either positive or negative, Eq. (1) has a most immediate minimum for negatively correlated members (assuming that bias and variance for these models stay constant). Indeed, algorithms searching for negative correlation patterns have been proposed in the literature (e.g. Liu and Xao, 1999) and are an active area of research in the field of information science, though they are not the goal of this study. Only in the case where the members of the ensemble are all mutually positively correlated is the MSE minimised by a null covariance term, and accuracy and diversity would be optimised simultaneously. But, in general, negatively correlated (and thus dependant) members minimising the error is an indication that diversity and accuracy need to be assessed in isolation. In other words, we cannot expect that an ensemble promoting diversity is highly skilled in accuracy.

The Latin expression pauci ex tanto numero is extracted from the De Bello Gallico (The Gallic Wars, book 7, chapter 88 ) by G. J. Caesar (100-44 BC), and refers to the battle of the Roman army against the Gauls. The complete citation reads "pauci ex tanto numero incolumes se in castra recipient" and that translates to "few [Gauls] from a large number returned safely back to the camps". We therefore more peacefully decided to take the first part of the citation to stress the fact that only a few from a multitude of models will be the ones that will make the ensemble result relevant and will metaphorically survive in the end.

The paper is structured as follows. In Sect. 2 the scopes are highlighted and the dataset and methodology are presented. In Sect. 3 we introduce a metric for detecting similarities beyond the overarching ones, and use this metric to quantify the level of redundancy of the dataset. Redundancy reduction is achieved by applying several techniques (Sect. 4), which serve the scope of identifying the minimum number of elements necessary to explain the variance of the observational data. Once the dimension of the minimum set is established, we apply a number of member selection criteria (Sect. 5). The methods of member selection have the purposes of identifying the members (or the weights) that (i) have poorly correlated errors (thus non-redundant) and (ii) whose ensemble mean is skilful in terms of accuracy and precision. Conclusions are drawn in Sect. 6.
To what extent does an ensemble of different models put together on the grounds of opportunity and convenience produce a better result? How can one quantify the information in multi-model ensembles that is necessary and relevant? Answers to these questions were already anticipated by Potempski and Galmarini (2009), where the angle of attack was more on whether the composition of the ensemble could be investigated a priori. A theoretical framework and conditions were indeed identified but cannot be put in practice for all cases. Solazzo et al. (2012a) clarified the necessity of a posterior screening of the data and heuristically identified a possible methodology. In this paper we analyse various techniques available to address the following issues:

1. Quantification of ensemble redundancy: i.e. the minimum set of members required to explain the variance of the observations.

2. Selection of members to reduce the ensemble redundancy: if two models, or their errors, are highly correlated, one can be expressed in terms of the other by a simple scaling factor. If many redundant models are combined together, there would be loss of valuable information due to dependant biases.

We investigate the correlation between errors produced by AQ models run by 12 groups in the context of the Air Quality Modelling Evaluation International Initiative (AQMEII) (Rao et al., 2011). For all of the analyses we use hourly time series for the months of June-July-August (JJA) 2006 of the gaseous species $\mathrm{O}_{3}, \mathrm{CO}, \mathrm{NO}_{2}$ and $\mathrm{SO}_{2}$. We apply the analysis to two distinct regions of Europe, which have been subjects of in-depth investigations in other AQMEII studies (Solazzo et al., 2012a, b, 2013; Vautard et al., 2012):

- Region $1(-10,5)^{\circ} \mathrm{W},(42,60)^{\circ} \mathrm{N}$, including the UK, France, northern Spain and Belgium;

- Region $2(5,24.5)^{\circ} \mathrm{W},(46,60)^{\circ} \mathrm{N}$, continental Europe, including Germany, Poland, Austria and the Czech Republic.

The modelled and observed time series have been spatially averaged over region 1 and 2 defined above. The number of receptors - by species - in each region is reported in Table 1 and the participating models are summarised in Table 2. Details about the model settings and operational evaluation against observational data can be found in Solazzo et al. (2012a, b, 2013) and Vautard et al. (2012), with the exception of the GEM-AQ model (Côté et al., 1998; Kaminski et al., 2008), which did not take part in the previous AQMEII analyses. The AQMEII ensemble of models forms a typical ensemble of opportunity, in which diverse AQ models and meteorological drivers are applied; emission and boundary 
Table 1. Number of rural receptors by species and regions.

\begin{tabular}{lrrrr}
\hline Europe & $\mathrm{O}_{3}$ & $\mathrm{SO}_{2}$ & $\mathrm{NO}_{2}$ & $\mathrm{CO}$ \\
\hline Region1 & 199 & 34 & 56 & 23 \\
Region2 & 225 & 131 & 136 & 54
\end{tabular}

conditions are, however, largely shared, making the distribution of model errors neither systematic nor random. The history of regional-scale modelling has also forcibly produced a number of common elements to all the models, which should be considered an a priori contaminating element of the ensemble results.

Since an accurate estimation of multivariate a PDF is hard to achieve due to the computational cost it entails even for a small number of models (Peng et al., 2005), we decide to focus on quantifying the amount of information two models share measured by the redundancy, which can be computed more easily. Given the output from two models, $\boldsymbol{x}$ and $\boldsymbol{y}$ organised as a two-column table - with $\operatorname{cov}_{x y}$ their covariance, and $p(\cdot)$ their joint PDF - the redundancy can be defined either through the redundancy index $\rho I(\boldsymbol{x}, \boldsymbol{x})$ (Stewart and Love, 1968), which is a metric for quantifying the portion of variance already being accounted for by other members of the ensemble (Eq. 2), or by the mutual information among models $I(\boldsymbol{x}, \boldsymbol{x})$ (Peng et al., 2005; Ding and Peng, 2005) (Eq. 3):

$\rho I(\boldsymbol{x}, \boldsymbol{y})=\frac{\operatorname{trace}\left(\operatorname{cov}_{x y} \operatorname{cov}_{y y}^{-1} \operatorname{cov}_{y x}\right)}{\operatorname{trace}\left(\operatorname{cov}_{x x}\right)}$,

$I(\boldsymbol{x}, \boldsymbol{y})=\iint p(\boldsymbol{x}, \boldsymbol{y}) \log \frac{p(\boldsymbol{x}, \boldsymbol{y})}{p(\boldsymbol{x}) p(\boldsymbol{y})} d \boldsymbol{x} d \boldsymbol{y}$.

Equation (2) is related to the prediction of $\boldsymbol{x}$ by $\boldsymbol{y}$ by multiple linear regression. $\rho I(\boldsymbol{x}, \boldsymbol{y})$ is a weighted average of the squared multiple correlation coefficient between all pairs of variables of $\boldsymbol{x}$ and $\boldsymbol{y}$. It is a measure of the quality of the prediction of $\boldsymbol{x}$ by $\boldsymbol{y}$ and represents the proportion of explained variance in the regression of $\boldsymbol{x}$ by $\boldsymbol{y}$ (see e.g. Youness and Saporta, 2010). In the case of $\boldsymbol{x}$ and $\boldsymbol{y}$ one-dimensional vectors $\rho I$ returns $R^{2}$, the squared correlation coefficient. The mutual information in Eq. (3) is more complex and involves the PDFs of multivariate variables. In practical terms, $I$ is the level of repetition of two datasets, and the PDFs are computed as the frequency of unique elements belonging to both $\boldsymbol{x}$ and $\boldsymbol{y}$. Details about the implementation of Eq. 3 are given in Peng et al. (2005) and Yoon and Kim (2009).

\section{A metric for model similarities and comparability of errors}

Common biases are difficult to detect, especially for AQ models, where the variance of the noise can be comparable with that of the signal (and particularly for low concentrations). The AQMEII database (Galmarini et al., 2012) includes results from members sharing meteorological drivers, emissions and chemical boundary conditions (Table 2). It was proven that these input fields introduce systematic biases in the model results (Solazzo et al., 2012a, b). A simple error metric would not be adequate to detect any type of underlying commonality other than these overarching biases. Therefore we need a metric that (a) explores hidden similarities, i.e. those underlying common modules and parameters in the model, and that (b) is robust enough to be used under a number of scenarios. Having in mind that no wonder metric exists, and that different metrics produce different results (Gleckler et al., 2008), we opted for the metric $d_{\mathrm{m}}$ proposed by Pennel and Reichler (2011) (hereafter referred to as PR2011), which explores the biases of models and removes from each model the dominating similarities, thus making individual model errors more dissimilar and unveiling "hidden" trends that are masked by overarching commonalities. Another choice would have been a metric accounting for the variance as element of model similarity, as it is related to model uncertainties. The variance, however, is more difficult to apply in practice as it would require sensitivity model simulations (e.g. Garaud and Mallet, 2011).

Let us start by defining the normalised deviation of models (mod) from observations (obs) as

$e_{i, \mathrm{~m}, \mathrm{~s}}=\frac{\bmod _{i, \mathrm{~m}, \mathrm{~s}}-\mathrm{obs}_{i, \mathrm{~s}}}{\sigma_{\mathrm{s}}}$,

where $\sigma_{\mathrm{s}}$ is the standard deviation of the observed chemical species $s, i=1, \ldots, N$ is the index of the time series, $\mathrm{m}$ is the model index and $s$ that of the species being considered $\left(\mathrm{O}_{3}, \mathrm{CO}, \mathrm{NO}_{2}, \mathrm{SO}_{2}\right)$. The normalisation in Eq. (4) makes the errors more comparable for different chemical species and units. We now define the MM-error pattern (MME) as

$\mathrm{MME}_{i, \mathrm{~s}}=\frac{1}{M} \sum_{m=1}^{M} e_{i, \mathrm{~m}, \mathrm{~s}}$,

which contains the "bulk" of bias among models. To eliminate the dominating model similarities, we remove the portion of MME associated with each individual model error. According to PR2011, the removal of the portion of MME relevant to an individual model can be accomplished by calculating $d_{\mathrm{m}}$, the difference between the model error and the weighted MME, with the weight being the correlation coefficient between the $m$-th model error and the $\operatorname{MME}\left(R_{\mathrm{m}, \mathrm{MME}}\right)$ :

$\boldsymbol{d}_{\mathrm{m}, \mathrm{s}}=\boldsymbol{e}_{\mathrm{m}, \mathrm{s}}^{*}-R_{\mathrm{m}, \mathrm{MME}} \cdot \boldsymbol{M} \boldsymbol{M} \boldsymbol{E}_{\mathrm{s}}^{*}$

where the "**" indicates standardised vectors, calculated, for each time series, by subtracting the corresponding mean value $\overline{e_{\mathrm{m}}}$ and dividing by the standard deviation $\sigma_{e_{\mathrm{m}}}$ (we have now get rid of the index $i$ for a more compact notation) (details are given in PR2011). The standardisation 
Table 2. Participating models and features.

\begin{tabular}{|c|c|c|c|c|c|c|}
\hline \multicolumn{3}{|c|}{ Model } & \multirow{2}{*}{$\begin{array}{l}\text { Grid } \\
\text { spacing } \\
(\mathrm{km})\end{array}$} & \multirow{2}{*}{$\begin{array}{l}\text { No. of vertical } \\
\text { layers }\end{array}$} & \multirow[t]{2}{*}{ Emissions } & \multirow[t]{2}{*}{ Chemical BC } \\
\hline Code & Met & AQ & & & & \\
\hline DK1 & MM5 & DEHM & 50 & $\begin{array}{l}29 \text { (top: } \\
100 \mathrm{hPa} \text { ) }\end{array}$ & $\begin{array}{l}\text { Global emission } \\
\text { databases, EMEP }\end{array}$ & Satellite measurements \\
\hline FR3 & MM5 & Polyphemus & 24 & 9 (top: $12 \mathrm{~km}$ ) & Standard $^{\mathrm{a}}$ & Standard \\
\hline HR1 & PARLAM-PS & EMEP & 50 & 20 & EMEP model & $\begin{array}{l}\text { From ECMWF and } \\
\text { forecasts }\end{array}$ \\
\hline UK2 & WRF & CMAQ & 18 & $\begin{array}{l}34 \text { (up to } \\
50 \mathrm{hPa} \text { ) }\end{array}$ & Standard $^{\mathrm{a}}$ & Standard \\
\hline DE2 & WRF & WRF/Chem & 22.5 & $\begin{array}{l}36 \text { (top: } \\
22.5 \mathrm{~km})\end{array}$ & Standard $^{\mathrm{a}}$ & Standard \\
\hline US4 & WRF & WRF/Chem & 22.5 & $\begin{array}{l}36 \text { (top: } \\
22.5 \mathrm{~km})\end{array}$ & Standard $^{\mathrm{a}}$ & Standard \\
\hline FI1 & ECMWF & SILAM & 24 & 9 (top: $10 \mathrm{~km}$ ) & $\begin{array}{l}\text { Standard anthropogenic } \\
\text { In-house biogenic }\end{array}$ & Standard \\
\hline FR4 & MM5 & Chimere & 25 & $\begin{array}{l}9 \text { (up to } \\
500 \mathrm{hPa})\end{array}$ & MEGAN, standard & Standard \\
\hline PL1 & GEM & GEM-AQ & $\begin{array}{l}0.2 \\
\text { degree }^{b}\end{array}$ & $\begin{array}{l}28 \text { (up to } \\
10 \mathrm{mb} \text { ) }\end{array}$ & $\begin{array}{l}\text { Standard over AQMEII } \\
\text { region; global } \\
\text { EDGAR/GEIA over } \\
\text { the rest of the global } \\
\text { domain }\end{array}$ & $\begin{array}{l}\text { Global variable grid } \\
\text { setup (no lateral } \\
\text { boundary conditions) }\end{array}$ \\
\hline NL1 & ECMWF & Lotos-EUROS & 25 & 4 (top: 25 km) & Standard $^{\mathrm{a}}$ & Standard \\
\hline DE1 & COSMO & Muscat & 24 & 40 (top: $24 \mathrm{~km}$ ) & Standard $^{\mathrm{a}}$ & Standard \\
\hline US3 & MM5 & CAMx & 15 & 20 (top: $24 \mathrm{~km}$ ) & MEGAN, standard & Standard \\
\hline DE3 & COSMO-CLM & CMAQ & 24 & $\begin{array}{l}30 \text { (up to } \\
100 \mathrm{hPa})\end{array}$ & Standard $^{\mathrm{a}}$ & Standard \\
\hline
\end{tabular}

a Standard anthropogenic emission and biogenic emission derived from meteorology (temperature and solar radiation) and land use distribution implemented in the meteorological driver (Guenther et al., 1994; Simpson et al., 1995).

b Corresponding to $22.2 \mathrm{~km}$ at the domain centre.

serves the purposes of making the results for different species inter-comparable (the correlation operator is bias and normalisation independent), and makes the correlation and covariance interchangeable. As mentioned above, removal of MME makes model errors more dissimilar and uncovers "hidden" trends that are outweighed by overarching commonalities. For example, $\operatorname{corr}\left(e_{\mathrm{FR} 3, \mathrm{O}_{3}}^{*}, e_{\mathrm{FI} 1, \mathrm{O}_{3}}^{*}\right)=0.73$, while $\operatorname{corr}\left(d_{\mathrm{FR} 3, \mathrm{O}_{3}}, d_{\mathrm{FI} 1, \mathrm{O}_{3}}\right)=0.36$. The subtraction of the correlated portion of the bulk error from the individual error emphasises the real differences among models. On the other hand, in the case of the same modelling system operated by different groups such as DE2 and US4, the correlation among $e_{i}^{*}$ is approximately the same as that among the $d_{i}$.

We provide two graphical examples of the efficacy of $d_{\mathrm{m}}$ vs. $e_{\mathrm{m}}$. The correlation between individual model error and the MME (corr $\left.\left(e_{i}, \mathrm{MME}\right)\right)$, averaged over all models, is reported in Fig. 1. The correlations are largely positive due to commonalities, and also show dependence on the region (correlations for $\mathrm{SO}_{2}$ are different over the two regions). In Fig. 1 the correlation $\operatorname{corr}\left(d_{i}, d_{j}\right)$ is also shown, averaged over all model pairs. The values for the curves for the two regions are very similar and small, indicating that the effect of MME has been largely mitigated. The removal of MME also makes the model errors region independent, as shown by the similar curves of $\operatorname{corr}\left(d_{i}, d_{j}\right)$. In Fig. 2 we report the associativity tree (the dendrogram; see details in Sect. 4.4) of $\operatorname{cov}\left(d_{i}, d_{j}\right)$ and $\operatorname{cov}\left(e_{i}, e_{j}\right)$ for the joint time series of the four pollutants in region 2 . While $e_{\mathrm{m}}$ associations are based on the species (model errors for each species are the most correlated), $d_{\mathrm{m}}$ associations are drastically diverse, and unexpected patterns emerge. Models are grouped by the bias underlying modules and/or parameters strictly associated with the physics and chemistry of a given compound. The diversity for $d_{\mathrm{m}}$ is higher with respect to the $e_{\mathrm{m}}$ dendrogram, and the number of disjoint clusters is at least six (distance level of $\sim 0.9$ ), while four $e_{\mathrm{m}}$ clusters were identified (at an even smaller distance of $\sim 0.7$ ). 


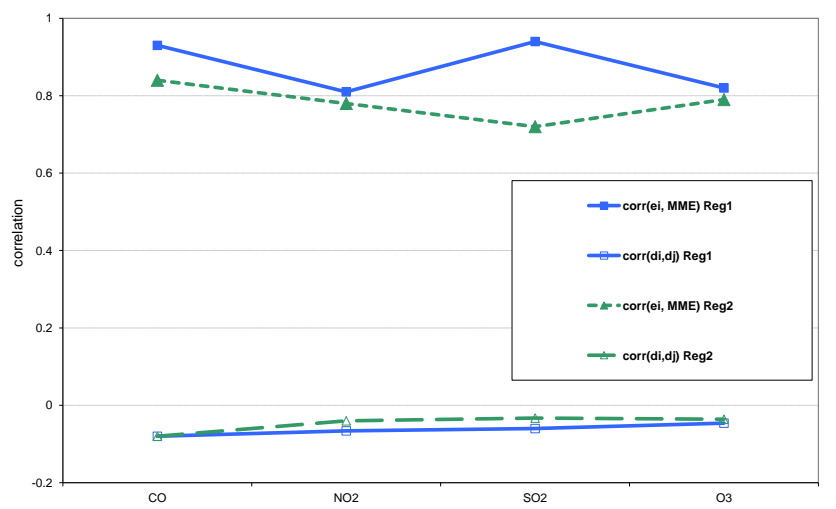

Fig. 1. Correlation of errors (between individual model and MME and between $d$ for all model pairs) for region 1 and region 2 of Europe for the months of JJA of 2006.

\section{Ensemble redundancy through error analysis}

In Fig. 3 the covariance $\operatorname{cov}\left(d_{i}, d_{j}\right)$ is shown for the species of the European region 2 (plots for region 1 are omitted for brevity). Mutual model covariance is indicated by the positioning of the model codes in black with respect to models on the horizontal axis. Because the covariance matrix is symmetric, we display only half of it for clarity. The model in red indicates the variance $\left(\operatorname{cov}\left(d_{i}, d_{i}\right)\right)$. In these plots we also report the following:

- The redundancy measured by $R^{2}$ (blue crosses), the square of $\operatorname{corr}\left(d_{i}, d_{j}\right) . R^{2}$ represents the amount of variance already explained by the regressor model and, for model pairs, corresponds to the redundancy index $\rho I$.

- The mutual information $I$ (vertical segments in orange).

Because of the normalisation of the metric $d_{\mathrm{m}}$, the covariance and redundancy can be expressed on the same scale, between -1 and 1 .

Depending on the pollutant, the mutual relationships among members vary greatly, proving that for AQ models many factors (chemistry and dispersion modules, meteorology, grid spacing) weigh the final outcome, as was also found to be the case for climate models (PR2011; Annan and Hargreaves, 2010). Overall, errors do not seem to co-vary more in the case of two instances of the same AQ model (DE3 and UK2 for example) than for different combination of meteodispersion models (FR4, DE1 for $\mathrm{O}_{3}$ and $\mathrm{CO}$; HR1 and UK2 for $\mathrm{SO}_{2}$ ). The sharing of routines specifically designed for certain pollutants and processes could offer a plausible explanation. It is often the case that model developers borrow entire model components as their use was demonstrated to be an improved, or sometimes the only, solution for simulating a process. For example, the ISORROPIA module (Nenes

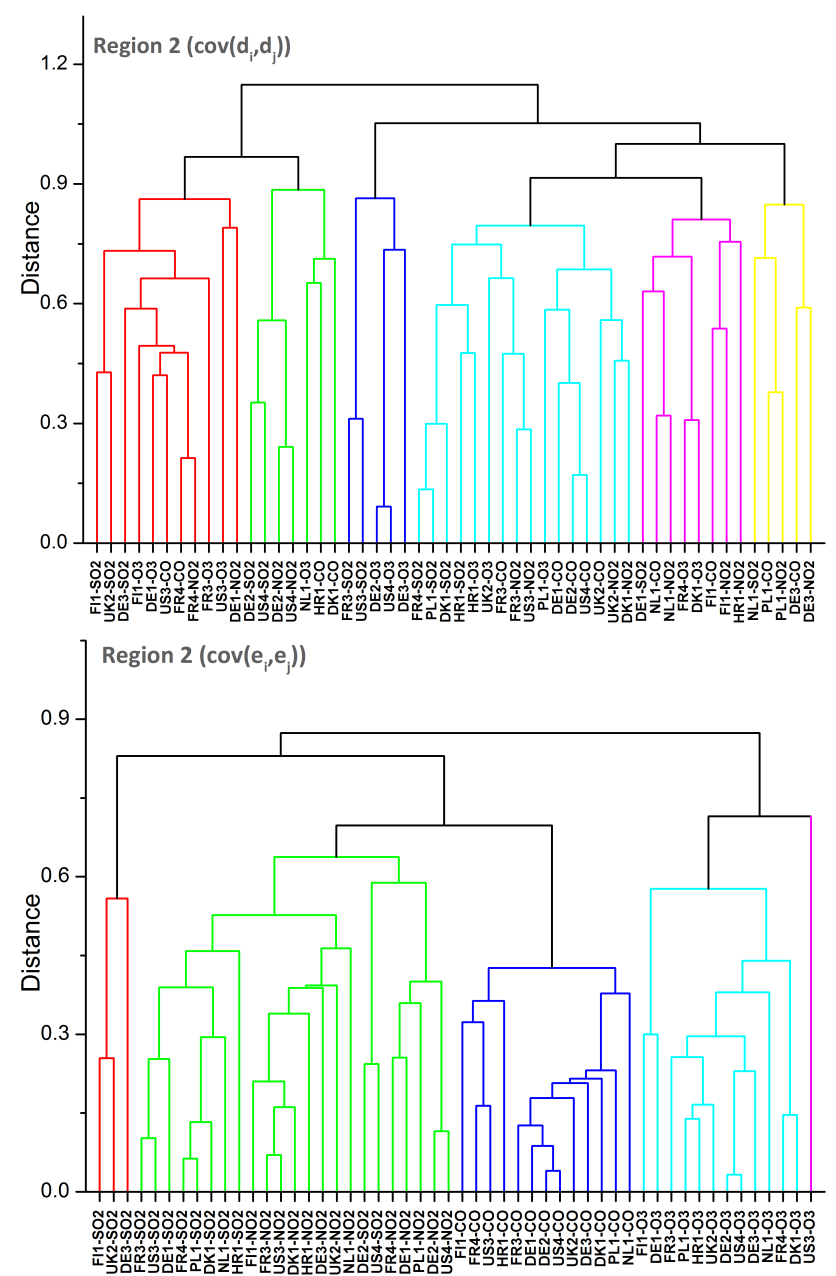

Fig. 2. Associativity trees for all models and species (European region 2) using (a) the $\operatorname{cov}\left(d_{i}, d_{j}\right)$ and (b) the $\operatorname{cov}\left(e_{i}, e_{j}\right)$ as distance matrix.

et al., 1998) for inorganic pollutants, the resistive scheme by Zhang et al. (2001) for dry deposition and the scavenging parameterisation for wet deposition are all examples of shared routines among the majority of the AQMEII models (see Table 1 of Solazzo et al., 2012b).

Because the redundancy measured by $R^{2}$ is simply the ratio of the squared covariance to the variance, models with a large spectrum of covariance are also the more redundant (DK1 and DE1 for $\mathrm{O}_{3}$; US3 and US4 for CO; DK1 and DE3 for $\mathrm{SO}_{2} ; \mathrm{NL} 1, \mathrm{DE} 2$, US4 for $\mathrm{NO}_{2}$ ). The redundancy measured by the mutual information is often in line with that of $R^{2}$, although in some cases higher values are estimated. For example, DE2 and US4 (same models run by different groups), as well as US3 for CO and $\mathrm{NO}_{2}$ and FR4 and PL1 for $\mathrm{SO}_{2}$, due to $I$ being calculated as a raw frequency count, whilst $R$ derives from a regression analysis.

Annan and Hargreaves (2010) have suggested a technique to assess the amount of spanned variability of the MM 


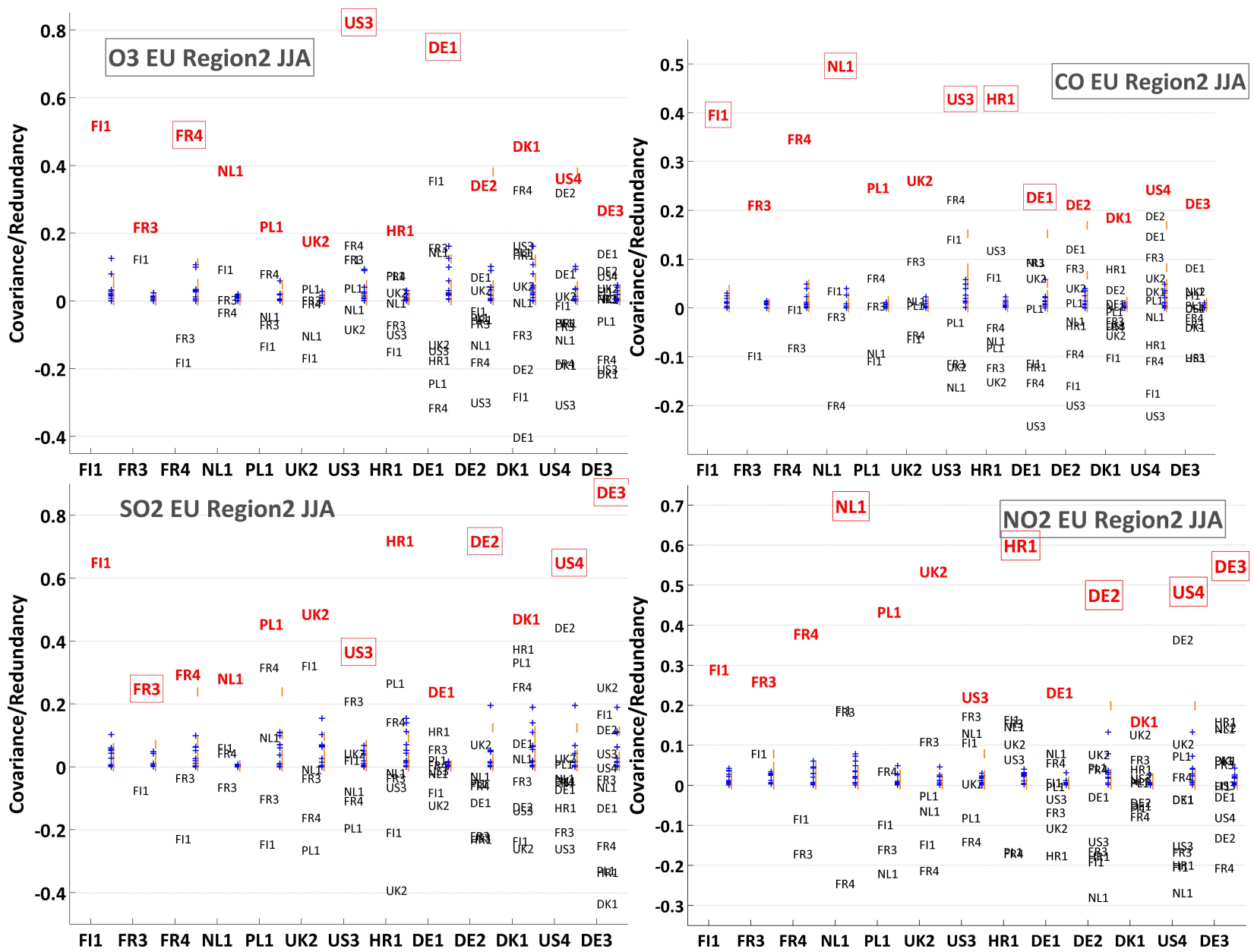

Fig. 3. $\operatorname{cov}\left(d_{i}, d_{j}\right)$ by species for European region 2 . In red the variance $\left(\operatorname{cov}\left(d_{i}, d_{j}\right)\right)$, in blue the range of redundancy $\left(\operatorname{corr}{ }^{2}\left(d_{i}, d_{j}\right)\right)$ and in orange the range of redundancy measured by means of the mutual information (see text).The models in the square are those whose ensemble mean produces the minimum MSE (see Sect. 5.5.1).

ensemble with respect to the observations, consisting in projecting the observation anomalies (the element-wise difference between the observations and their mean) onto the principal components (PCs) of the covariance matrix of the deviation of the ensemble of models from the MM mean (the element-wise difference between each model realisation and the MM ensemble mean). When applying this method to the AQMEII ensemble we find that just the first (or the first two for $\mathrm{O}_{3}$ ) component already exceeds the observed variance. When all components are taken into account, it results that the MM mean for the EU region 1 (region 2) can explain as much as 1.2 (1.7), 2 (4.8), 2.1 (9) and 7 (18) times of the observed variability for $\mathrm{O}_{3}, \mathrm{CO}, \mathrm{SO}_{2}$ and $\mathrm{NO}_{2}$, respectively (the large difference between region 1 and 2 for $\mathrm{NO}_{2}$ and $\mathrm{SO}_{2}$ is due to the much smaller variance of the observed values of these two compounds in region $2(\sim 4$ and 12 times smaller for $\mathrm{NO}_{2}$ and $\mathrm{SO}_{2}$, respectively)). According to the definition of Annan and Hargreaves (2010), the ensemble is therefore wide. A wide ensemble can be interpreted also in terms of lack of reliability with a rank histogram (Talagrand et al., 1998) exhibiting a "central dome" pattern: the ensemble performs poorly in predicting less frequent episodes (both high and low concentrations) and lacks sharpness. Given the massive application of AQ models in regulatory applications and the more and more stringent AQ targets, the detected overconfidence can have considerable cost. Dealing with a wide ensemble implies that there is a substantial amount of redundant variability, i.e. variability already accounted for by other models, which is often the case in an ensemble of opportunity. One consequence of this is that not all information contained in the ensemble is needed in principle. In particular, that would be the case if the presence of redundant information were to produce a deterioration of the ensemble result, as investigated in the next section. One plausible explanation is that the ensemble size, constrained by the available members, is simply too large. 


\section{Quantifying ensemble redundancy through dimensionality}

The immediate advantage of reducing the dimensionality by discarding redundant information is the reduced computational costs and noise. Data mining and reduction are active areas of research in various fields, from genetics to ecology to machine learning. There exist a plethora of methods aiming at detecting commonalities, most of which developed ad hoc for specific applications, such as independent component analysis (Kong et al., 2008); maximum relevance, minimum redundancy (Peng at al., 2005); the methods reviewed by Grömping et al. (2007); and others. However, it is seldom the case that a method passes the barriers of its developing community to be adopted in a field other than the original one.

Here we explore some analytical dimension-reduction techniques proposed in various permutations in the climate modelling community, whose outcome is exclusively the dimension of the subspace. Selecting the members belonging to that subspace is a different problem, and is addressed in Sect. 5.

\subsection{Eigenvalue methods}

We calculated the effective number of models (also known as the effective number of degrees of freedom) sufficient to reproduce the variability of the full ensemble (the MM ensemble generated with all available members) as

$$
M_{\mathrm{eff}}=\frac{\left(\sum_{k=1}^{M} \lambda_{k}\right)^{2}}{\sum_{k=1}^{M} \lambda_{k}^{2}},
$$

with $\lambda$ eigenvalue of the $\operatorname{corr}\left(d_{i}, d_{j}\right)$ matrix. Theoretical derivation of Eq. (7) can be found in Bretherton et al. (1999). Under the assumption that the modelled and observed fields are normally distributed, the fraction of the overall variance expressed by the first $M_{\text {eff }}$ eigenvalues is of $86 \%$ (Eq. (8) of Bretherton et al., 1999).

The sum over all eigenvalues at the nominator of Eq. (7) expresses all the variability that is attainable in an $M$ dimensional vectorial base of orthogonal vectors. By construction, $\sum_{k=1}^{M} \lambda_{k}=M$, and only if all eigenvalues were equal to unity would Eq. (7) return $M_{\text {eff }}=M$; that is, all directions are equally important. In reality there exist eigenvalues that are larger than unity, and consequently others that are less than unity, and since these are squared (denominator of Eq. 7), the contribution of the former outweighs that of the latter so that $M_{\text {eff }}<M$ approximately in the amount of the number of eigenvalues larger than unity (Guttman (1954) and Kaiser (1960) indeed proposed to adopt this as a rule for determining the number of factors to retain, supposing that it
Table 3. $M_{\text {eff }}$ from Eq. (6). Values have been calculated using $\operatorname{corr}\left(d_{i}, d_{j}\right)\left(\operatorname{corr}\left(e_{i}, e_{j}\right)\right)$.

\begin{tabular}{lrrrr}
\hline Europe & $\mathrm{O}_{3}$ & $\mathrm{SO}_{2}$ & $\mathrm{NO}_{2}$ & $\mathrm{CO}$ \\
\hline Region1 & $5.8(2.3)$ & $5.7(1.3)$ & $6.5(2.2)$ & $6.5(1.3)$ \\
Region2 & $5.2(2.5)$ & $5.3(3.2)$ & $5.9(2.5)$ & $5.6(1.9)$ \\
\hline
\end{tabular}

makes no sense to retain components that explain less variance than the original standardised variables). Thus, we can replicate the variability of the $M$ members by an $M_{\text {eff }}$ dimensional subset in a vectorial space whose base is generated by the eigenvectors of the leading eigenvalues. On the other hand, if all error fields were similar, only one eigenvalue would be non-zero, and $M_{\text {eff }}=1$.

By applying Eq. (7) to the datasets of model errors ( $\operatorname{corr}\left(d_{i}, d_{j}\right)$ ), we find that $M_{\text {eff }}$ is in the range 5 to 6.5 . If the MME term is retained (that is, $M_{\text {eff }}$ is calculated from $\left.\operatorname{corr}\left(e_{i}, e_{j}\right)\right)$, we find much lower values for $M_{\text {eff }}$ as consequence of most of the similarity among models being expressed by the MME term (Table 3).

\subsection{Principal components analysis (PCA)}

Principal components analysis (PCA) (Jolliffe, 2002) is probably the most well known and widespread unsupervised dimension-reduction technique. It is based on eigenanalysis to select uncorrelated directions associated with the largest variances. Relationships between PCA and clustering (Ding and He, 2004), redundancy (Jolliffe, 2002), multidimensional scaling (Groenen and van de Velden, 2004) and regression analysis (Jong and Kotz, 1999) have been documented, proving the versatility of this method. For example, the ratio of the sum of leading eigenvalues to the sum of all eigenvalues obtained by means of PCA is proportional to the ratio of the regression sum of squares $\left(\mathrm{SS}_{\mathrm{reg}}\right.$ ) (explained or signal variance) and the total sum of squares $\left(\mathrm{SS}_{\text {tot }}\right)$ (the total variance) in regression analysis. This latter ratio is the coefficient of determination $R^{2}$, the redundancy index (Jun et al., 2008).

Equation (7) provides an analytical estimate of the dimensionality of the subspace of models to produce the information of the whole ensemble. Graphically, the "scree test" (Cattell, 1966) is often applied in problems of dimension reduction. First we produce a plot of the number of dimensions vs. quantities related to the amount of variability or independence, measured by appropriate metrics, and then we use the "elbow criterion" by seeking the point at which the curve levels off to a plateau. To produce a scree plot from Eq. (7), we look at $M_{\text {eff }}$ as a dependent variable of the number of models. Curves are reported in Fig. 4 for the four pollutants and the two European regions. The variability scale is calculated as the cumulative variability. The two sets of curves have been derived from $\operatorname{corr}\left(d_{i}, d_{j}\right)$ and from $\operatorname{corr}\left(e_{i}, e_{j}\right)$. We 

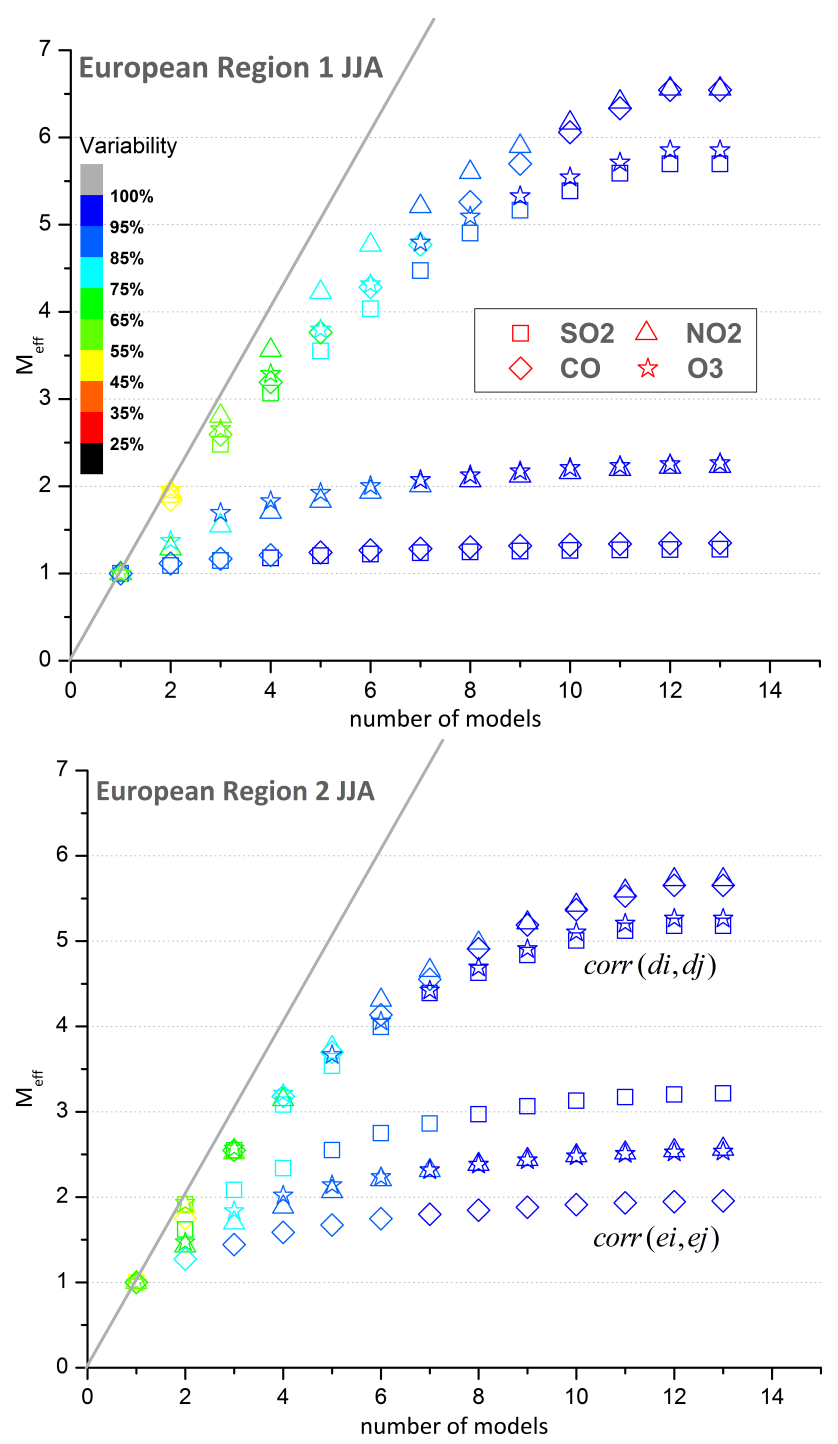

Fig. 4. $M_{\text {eff }}$ (Eq. 7) as function of the number of models for EU region 1 and region 2 . The two sets of curves have been generated from the $\operatorname{corr}\left(d_{i}, d_{j}\right)$ (top curves) and the $\operatorname{corr}\left(e_{i}, e_{j}\right)$ (lower curves) matrixes. The cumulative variability is colour coded. In grey is the one-to-one line.

notice that in both sub-regions $M_{\text {eff }}$ from $\operatorname{corr}\left(e_{i}, e_{j}\right)$ is much lower and that variability above $80 \%$ is reached by the first 2-3 leading eigenvalues. As noted by PR2011, the concavity of the curves over the number of models indicates that the addition of more models to the ensemble is not compensated by a linear increase in the overall information. This is a straight consequence of commonalities among members: chances that a new member shares features with an existing one increases as the ensemble size does. This would not happen in the case of independent models.

\subsection{Multi-dimensional scaling (MDS)}

Another method to create a scree plot is to use a multidimensional scaling (MDS) algorithm (Borg and Groenen, 2005) for determining the relationships between model errors. MDS searches for a spatial configuration of the objects such that the Euclidean distance (for which given two points $\boldsymbol{P}\left(p_{1}, p_{2} \ldots\right)$ and $\boldsymbol{Q}\left(q_{1}, q_{2}, \ldots\right)$, their distance is calculated as $\left.\sqrt{\left.\sum\left(p_{i}-q_{i}\right)^{2}\right)}\right)$ among them matches their proximities as closely as possible. Here, we use the $\operatorname{corr}\left(d_{i}, d_{j}\right)$ matrix as proximities. The degree of correspondence between the distances among points implied by MDS map and the input matrix is measured by a stress function, the minimisation of which also provides information about the dimensionality of the subspace covering the whole variability of the data. Avoiding detailing too much, in MDS theory the Euclidean distance $s_{i j}$ between two rows of a matrix $\mathbf{X}$ is defined as

$s_{i j}=\left(\sum_{k=1}^{p}\left(x_{i k}-x_{j k}\right)^{2}\right)^{1 / 2}$.

The objective of MDS is to find the elements of $\mathbf{X}$ minimising the difference between $s_{i j}$ and $d_{i j}$ (the elements of the proximity matrix $\left.\operatorname{corr}\left(d_{i}, d_{j}\right)\right)$ :

$\sigma^{2}(\mathbf{X})=\sum_{i=2}^{n} \sum_{j=1}^{i-1}\left(d_{i j}-s_{i j}(\mathbf{X})\right)^{2}$,

where $\sigma^{2}$ is the raw stress function. Minimisation of the stress function is not trivial, and thus numerical iterative methods are employed (Borg and Groenen, 2005). By running the minimisation problem for different values of $p$ in Eq. (9), we plot the stress against the dimension. Results for the European region 2 are reported in Fig. 5 (results for region 1 are very similar and therefore not shown). The "elbow" in the scree plot indicates when more dimensions only yield a negligible improvement in terms of stress. The trend of the curves in Fig. 5 (similar for all pollutants) indicates four as the number of independent components that best fit the data, i.e. about one-third of the whole sample size.

\subsection{Hierarchical clustering (HC)}

Given a dataset of $M$ instances $\mathbf{X}=\left\{X_{1}, X_{2}, \ldots, X_{M}\right\}$, a clustering algorithm generates $r$ disjoint clusters based on a distance metric, represented as $\Pi=\left\{\pi^{1}, \pi^{2}, \ldots, \pi^{r}\right\}$. Each clustering solution $\pi^{i}$ is a partition of the dataset $\mathbf{X}$ into $\mathrm{K}^{i}(i=1, \ldots, r)$ disjoint clusters of instances, represented as $\pi^{i}=\left\{c_{1}^{i}, c_{2}^{i} \ldots c_{K^{i}}^{i}\right\}$, where $\bigcup_{k} c_{k}^{i}=\mathbf{X}$ (Fern and Brodley, 2004). A typical output of $\mathrm{HC}$ is a dendrogram or associativity tree, where redundant models are grouped together and the level of similarity among groups is based on the distance between the elements of the input matrix. Here, we use the standard Euclidean distance and the $\operatorname{corr}\left(d_{i}, d_{j}\right)$ as 


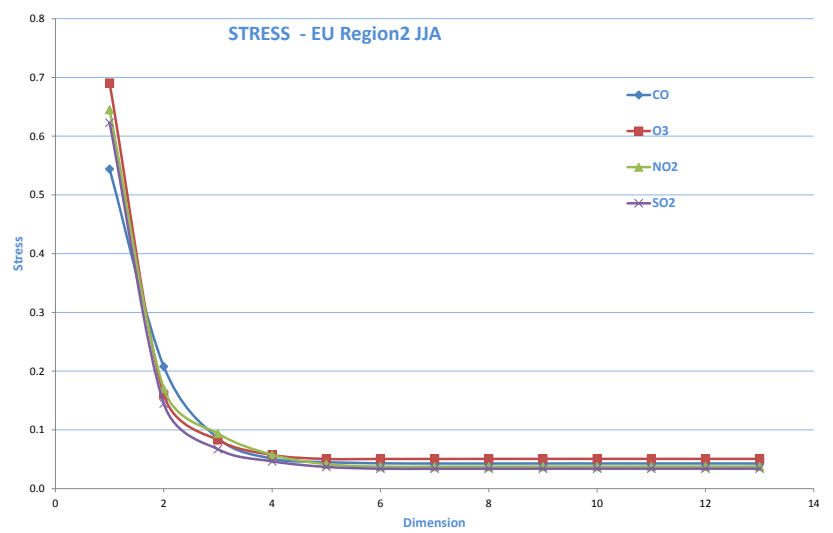

Fig. 5. Subspace dimension calculated by minimising the stress function in the MDS methodology. The $\operatorname{corr}\left(d_{i}, d_{j}\right)$ matrix is used as similarity criteria.

input matrix. Applications of $\mathrm{HC}$ and dendrogram representation for AQ ensemble modelling are documented in Riccio et al. (2012) and Solazzo et al. (2012a).

A fundamental challenge of the HC method is the high sensitivity to the controlling options (the agglomerative method, the distance metric, the number of clusters and the cut-off distance) that need to be determined case by case (Fern and Brodley, 2004). In particular, the cut-off (the threshold similarity above which clusters are to be considered disjointed) determines the dimension of the subspace of non-redundant models, and is typically decided by visual inspection of the dendrogram. After numerous tests, in this study the unweighted pair-group average was selected as the agglomeration method (Murtagh, 1984), with the cut-off value set between 0.10 and 0.15 ( 1 being the maximum similarity) for all pollutants in both regions, which produced five disjointed clusters (Fig. 6) for all species. The cut-off value is chosen by looking at the structure of the dendrogram: it is convenient to break structures that are obviously disjointed, and within each structure, avoid separating highly connected groups, or groups of only two models. Common practice suggests cutting the dendrogram at the height where the distance from the next clustered groups is relatively large, and the retained number of clusters is small compared to the original number of models (Riccio et al., 2012). Looking at the dendrogram for ozone, for example, the two main branches at the top further split into two more at a relatively low similarity level, suggesting a plausible way to proceed. At an $\sim 10$ to $15 \%$ similarity level, five clusters are detected for all species in both regions.

\subsection{Comparing the different methods: discussion}

Given the normalisation implied by the metric $d_{\mathrm{m}}$, we found $M_{\text {eff }}$ to range between $5.2\left(\mathrm{O}_{3}\right.$ in region 2$)$ and 6, with only $\mathrm{NO}_{2}$ and $\mathrm{CO}$ in region 1 requiring 6.5 components (Table 3 ).

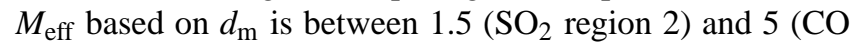

region 1) times higher than the values based on $e_{\mathrm{m}}$ (values in parenthesis in Table 3). The variability of $M_{\text {eff }}$ among species depends on the heterogeneity of processes and sources within the two regions, as well as on the receptors coverage. Despite having removed the commonalities among models through the MME, we still found a level of redundancy above $50 \%$, being $M_{\text {eff }}$ less than half of the size of sample.

Results of $\mathrm{HC}$ analysis indicates that at an $\sim 15 \%$ similarity level, five clusters are detected. The between-class variance (weighted average of the mean distance of each cluster and the mean distance of the whole dendrogram) detected by the five components generated by the HC method is between 70 and $80 \%$ of the total variance (depending on the variable), which would be fully reproduced only in the case of a cut-off level at the root of the dendrogram tree (one cluster only). On the other hand, the within-class variance (average distance within each cluster) is an estimate of the redundancy, as it is proportional to the cluster-averaged coefficient of determination $R^{2}$ (Moesa et al., 2005). This result is in line with that obtained by applying PCA: $M_{\text {eff }}$ in that case explained $86 \%$ of the total variance (Sect. 4.2) with a slightly larger number of models. Thus the two techniques are consistent for similar amount of variance. Dimensionality through MDS and the minimisation of the stress function has returned a number of components of four. In general though, MDS fit indexes are descriptive and do not always provide an absolute criterion for selecting the best dimensionality (Tinsley and Brown, 2000).

To summarise, the ensemble of models is highly redundant even after having removed the MM error. It is possible to reduce the full datasets of more than 50\%, down to 5-6 components. As discussed next, this allows for reducing noise and improving accuracy. The methods adopted give consistent results, and the one based on Eq. (7) seems the most reliable although quantifying the redundancy of the bias proved problem specific.

\section{Identifying the members of the reduced ensembles}

As many as $\sum_{i=1, m}\left(\begin{array}{c}M \\ i\end{array}\right)$ subspaces with dimension smaller than $m$ are identified by the $M$ members. It is therefore difficult to univocally identify a subset of members systematically outscoring all the others for a large number of skills (Garaud and Mallet, 2011). Ideally, once a skill or feature is identified, one could select the best-performing ensemble by extracting the $m$ best members. However, combinations of individually good models do not necessarily produce a good ensemble for a given feature: the $m$ best models are not necessarily the best $m$ (Cover, 1974) (further discussed in Sect. 5.5.1).

Countless methods for data reduction and member selection/weighting techniques have been developed by different communities, testifying that available methods are "fit for 

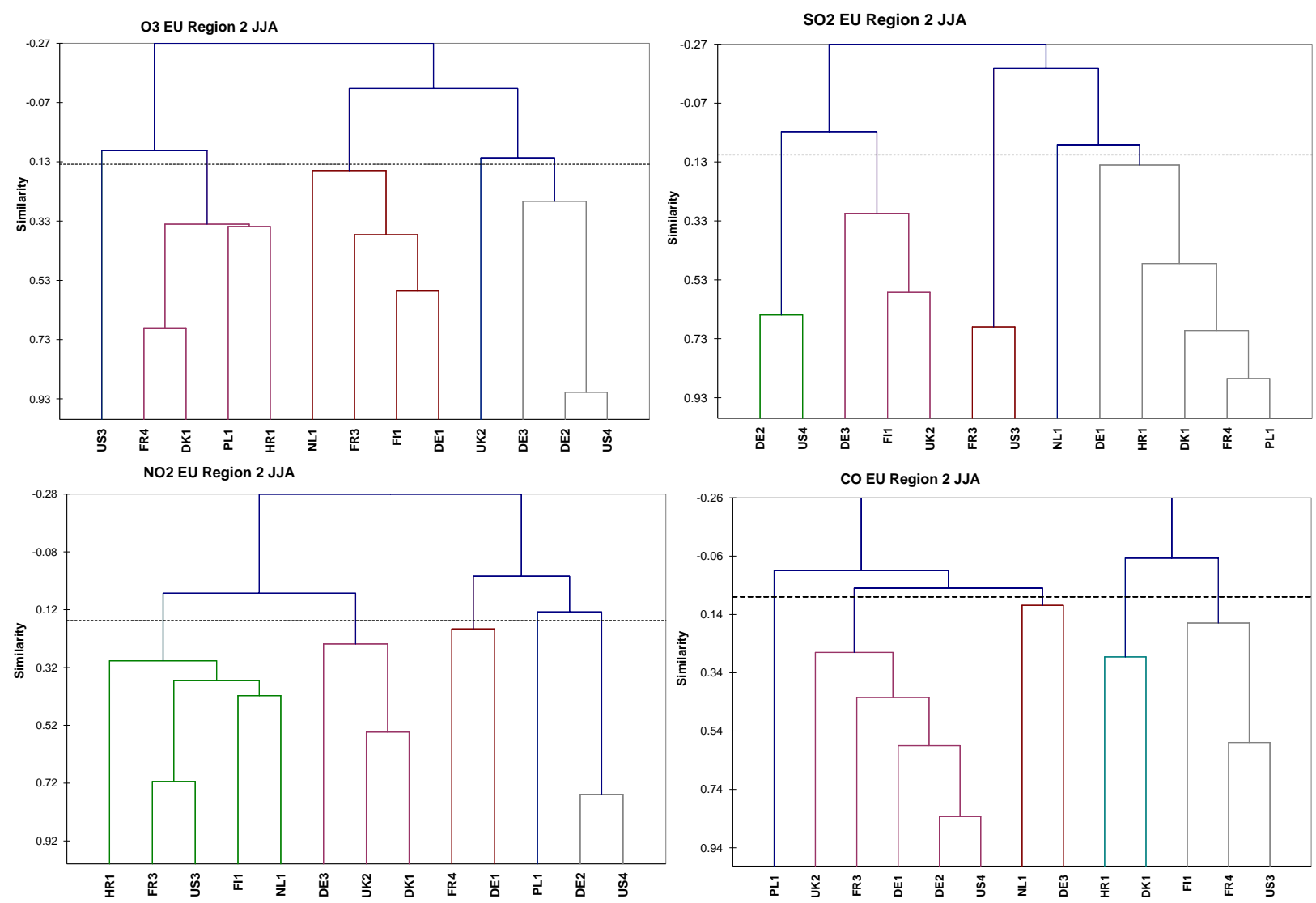

Fig. 6. Hierarchical clustering of $\operatorname{corr}\left(d_{i}, d_{j}\right)$ for EU region 2 . The dotted horizontal line defines the level of similarity. Disjoint clusters are identified by different colours.

purpose" rather than of general applicability. Here we exploit those and other methods for member selection and compare the reduced ensembles they produce to the full ensemble mean, taken as a benchmark. The techniques applied are as follows:

- Hierarchical clustering (HC).

- Multi-dimensional scaling (MDS).

- Minimisation of the root-mean-square error (minRMSE).

- Principal component analysis (PCA);

- Correlation-adjusted (marginal) correlation (CAR).

Not all of the methods above take into account the redundancy of members. The first two (HC and MDS) provide ensembles of less redundant members; the minRMSE technique is a heuristic method based on the minimisation of the error, and thus selection is skill driven (Solazzo et al., 2012a; Riccio et al., 2012; Knutti et al., 2010); PCA provides weights to the models along the directions of maximum variance; and finally, CAR is a score-based member selection method developed by Zuber and Strimmer (2011) that is hybrid of marginal correlation and regression analysis, and is shortly discussed in Sect. 5.5.1.

\subsection{Hierarchical clustering (HC)}

With reference to Fig. 6, members from each cluster are selected according to the individual model scores for bias, which is the metric underlying $d_{\mathrm{m}}$. The model ranked best for bias, among the models of each cluster, was the one selected to represent the cluster. The selected members are reported in Table 4. Other options have also been tested, as, for example, selecting the model closer to the centre of each cluster, or the models minimising the RMSE with respect to the cluster centroid. However, the reduced ensembles generated with these selection criteria were outperformed by that of members of minimum bias (see Sect. 5.5.2), and are therefore not shown.

\subsection{Multi-dimensional scaling (MDS)}

In MDS the distance among models can be used as proxy for independence, providing the visual aid needed for interpreting the grouping and selecting the most diverse member. MDS transforms the correlation between members into 
Table 4. Representative models ( $\operatorname{corr}\left(d_{i}, d_{j}\right)$ for the months of JJA). The number in parenthesis is the redundancy index $\rho I$ for each ensemble.

\begin{tabular}{|c|c|c|c|c|}
\hline \multicolumn{5}{|c|}{ EU region 1} \\
\hline & MDS & $\mathrm{HC}$ (min bias) & MinRMSE & CAR \\
\hline $\mathrm{O}_{3}$ & DE2;NL1;DK1;DE1 (0.27) & FI1,FR3,FR4,UK2,US4 (0.12) & FR4,PL1,US3,DE1,DK1 (0.53) & PL1,US3,HR1,UK2 (0.07) \\
\hline $\mathrm{SO}_{2}$ & US4;US3;FI1;NL1 (0.29) & FI1,FR3,DE1,HR1,US4 (0.22) & HR1,DE1 (FI1, UK2) (0.007) & FR3,DK1,FR4,UK2 (0.15) \\
\hline $\mathrm{CO}$ & DK1;FR3;HR1;US3 (0.25) & FI1,FR3,DE1,DK1,HR1 (0.17) & FI1,DE1 (NL1, US3) (0.02) & FR4,UK2,FI1,US4 (0.29) \\
\hline $\mathrm{NO}_{2}$ & FR4;FR3;PL1;DK1 (0.27) & FI1,FR3,DE1,DE2,HR1 (0.21) & FI1,UK2,US4 (DE2) (0.13) & UK2,FI1,DE3,HR1 (0.15) \\
\hline \multicolumn{5}{|c|}{ EU region 2} \\
\hline $\mathrm{O}_{3}$ & US4;US3;FI1;HR1 (0.31) & FI1;FR4;UK2;US3;DE3 (0.30) & FR4;US3;DE1 (FI1) (0.23) & DE1,US3,PL1,DE2 (0.35) \\
\hline $\mathrm{SO}_{2}$ & DK1;UK2;US4;FR3 (0.29) & DE3;US3;DE1;NL1;US4 (0.28) & DE3,FR3,US3,US4,DE2 (0.47) & DE3,DK1,UK2,NL1 (0.45) \\
\hline $\mathrm{CO}$ & US3;DK1;DE1;NL1 (0.60) & FI1;DE1;NL1;PL1;HR1 (0.15) & FI1,NL1,US3,HR1,DE1 (0.59) & UK2,DE3,HR1,NL1 (0.20) \\
\hline $\mathrm{NO}_{2}$ & US4;FI1;FR4;HR1 (0.29) & US3;DE1;PL1;DK1;DE2 (0.18) & NL1,US4,HR1,DE2,DE3 (0.55) & UK2,DE3,DE1,NL1 (0.08) \\
\hline
\end{tabular}

a distance, allowing a visual inspection of the mutual model positioning into a two-dimensional plane. The distance among members is the only information this methodology offers. Application of MDS for member selection in climate ensemble modelling can be found in Jun et al. (2008); the model space of Abramowitz (2010) is an extension of MDS, where the observations are treated as a de facto model. Figure 7 summarises the mutual model distance for the European region 2 .

\subsection{Minimum error (minRMSE)}

Solazzo et al. (2012a) show that the ensemble mean minimising the RMSE has also superior skills with respect to the full ensemble, both in terms of accuracy (error) and precision (variance). Application of this analysis yields (i) the number of dimensions to retain (the dimension of the subset) and (ii) the members to retain (the component of the subset, reported in Table 4). Knutti et al. (2010) and Annan and Hargreaves (2010) also explained the behaviour of the curves of RMSE obtained by randomly sampling the ensemble of members. In particular, the mean of the RMSE distribution decays proportionally to $\sigma_{\mathrm{obs}}(1+1 / m)^{0.5}$, as in the present study, is an indication that observations and model results are extracted from distributions with the same variance (the authors refer to this case as exchangeable or indistinguishable ensembles). Moreover, the fact that the RMSE, no matter how large $M$ is, can never reach zero is a consequence of the variability affecting the observations (from the error decomposition relationship, the variance of the observation is the lower bound for the error). Plot of RMSE for $\mathrm{O}_{3}$ (region 2) of the mean of random subsets of the ensemble members, plotted as function of subset size, is reported in Figure 8 (for brevity, plots for the other species are omitted). The curves show the maximum, mean and minimum of RMSE. The dash-dotted curve decays as $m^{-0.5}$ that would be the trend if the model errors were independent (Knutti et al., 2010; Annan and Hargreaves, 2010). We find a minimum for $m=3$, for which the RMSE is $\sim 37 \%$ smaller than the full ensemble mean. Adding more members to the ensemble increases the noise and deteriorates the accuracy. This would not happen if the model errors were independent as the curve in that case would decay monotonically.

\subsection{Principal components analysis (PCA)}

Although PCA cannot be applied for selecting individual, independent members, it can be nonetheless used to generate an artificial time series $\bmod _{\mathrm{PC}}$ obtained by projecting the original data onto the leading PCs. This generates a weighted ensemble, the weights being the projections of the model components onto the eigenvectors associated with the leading $m$ eigenvalues. We have applied PCA to the matrix of covariance $\operatorname{cov}\left(d_{i}, d_{j}\right)$ to disclose redundancy patterns (see Sect. 4.2). The reduced matrix $\mathbf{d}_{\mathrm{m} \text {,red }}$ is obtained by projecting $d_{\mathrm{m}}$ onto $\mathrm{PC}_{\mathrm{m}}$, the subspace of the first $m$ eigenvectors; that is, if $\Pi_{\mathrm{PCm}}$ denotes the projection operator on the subspace $\mathrm{PC}_{\mathrm{m}}$, then

$\mathbf{d}_{\mathrm{m}, \mathrm{red}}=\operatorname{PCm}\left(\mathbf{d}_{\mathrm{m}}\right)$.

We have discussed the scree plot of Eq. (10) (Sect. 4.2) and the dimensionality of the subspace $\mathrm{PC}_{\mathrm{m}}$. Ideally now we should be in a position to score the weighted ensemble obtained by retaining $m$ components. Getting the time series back from Eq. (10) is not trivial though, since Eq. (6) is a composite metric, and no similar applications of PCA have been found in the literature. The reduced time series is given by

$\boldsymbol{m o d}_{\mathrm{PC}}=\sigma_{\mathrm{obs}}\left(\sigma_{e_{\mathrm{m}}}\left(\mathbf{d}_{\mathrm{m}, \mathrm{red}}+\mathbf{R} \cdot \mathbf{M M} \mathbf{E}^{*}\right)+\overline{\boldsymbol{e}_{\mathrm{m}}}\right)+\boldsymbol{o b} \boldsymbol{s}$.

Some assumptions are necessary, as, for example, how to obtain the elements of MME and $\overline{\boldsymbol{e}_{\mathrm{m}}}$ (the mean error of each model) for the reduced space. The assumption we made consists in projecting these quantities onto $\mathrm{PC}_{\mathrm{m}}$ too, as it is not possible to associate them with their original time series.

The use of the observational data for recreating the time series is a major shortcoming of this methodology, which 


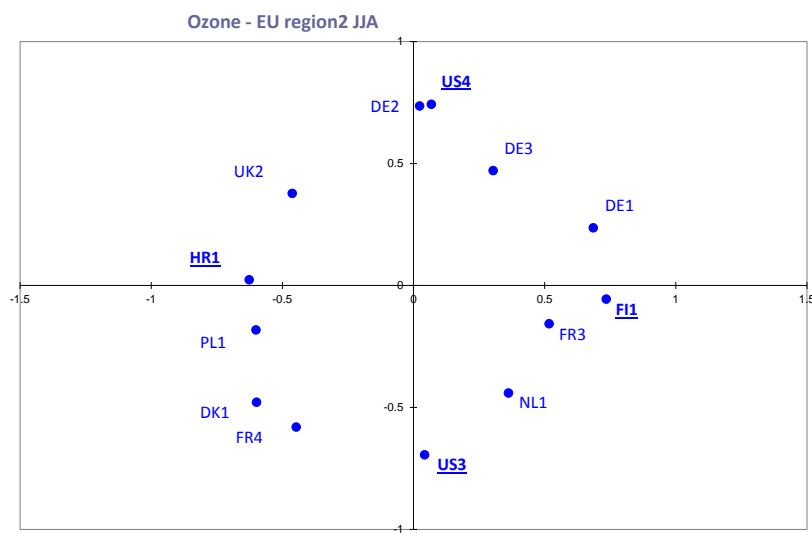

NO2 EU Region 2 JJA

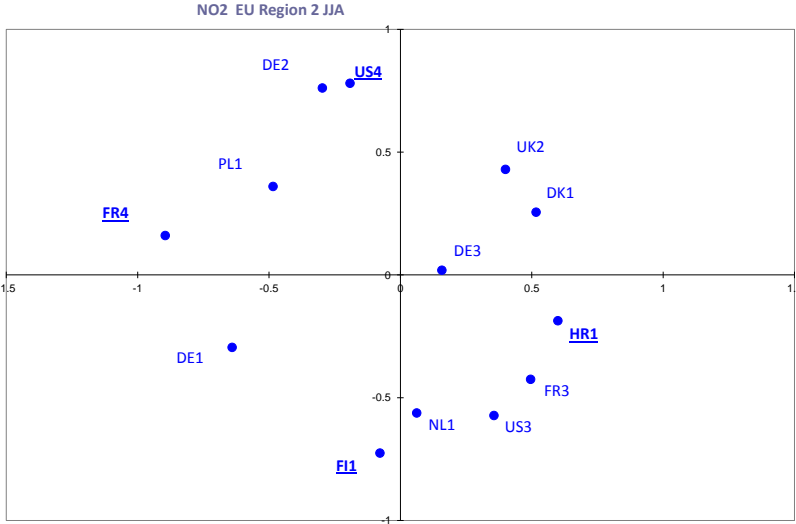

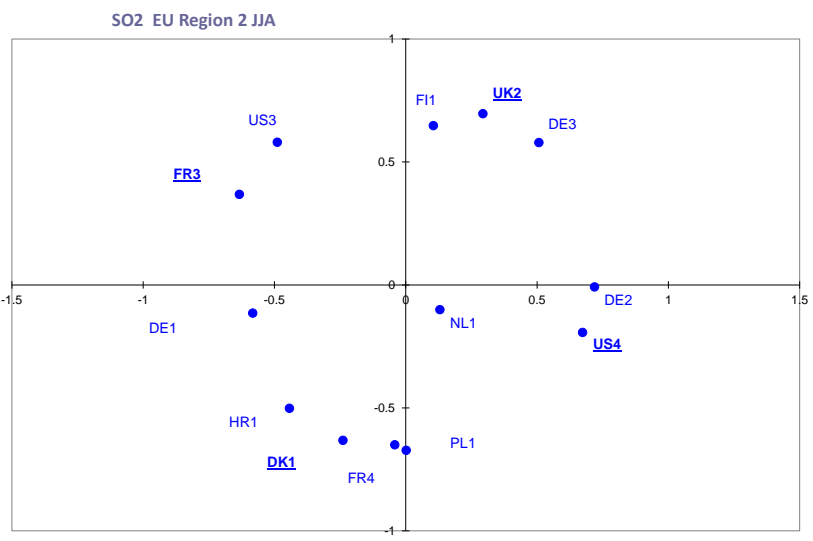

CO EU Region 2 JJA

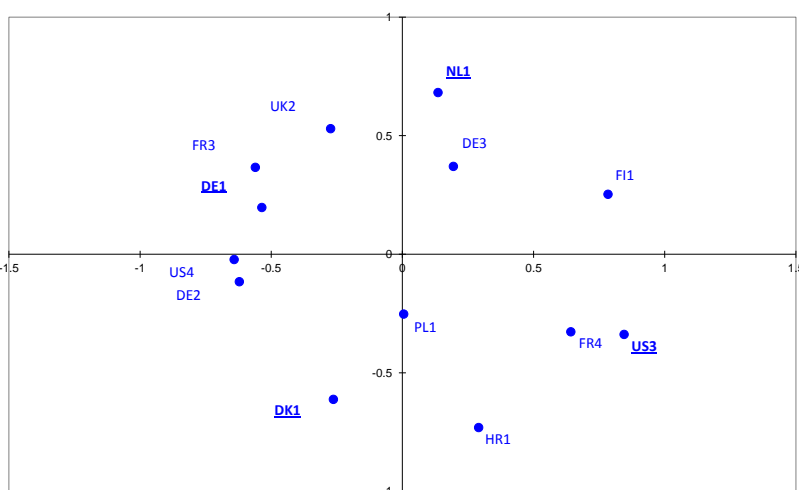

Fig. 7. 2-D MDS of $\operatorname{corr}\left(d_{i}, d_{j}\right)$ for EU region 2. Models underlined are those selected to generate the reduced ensemble.

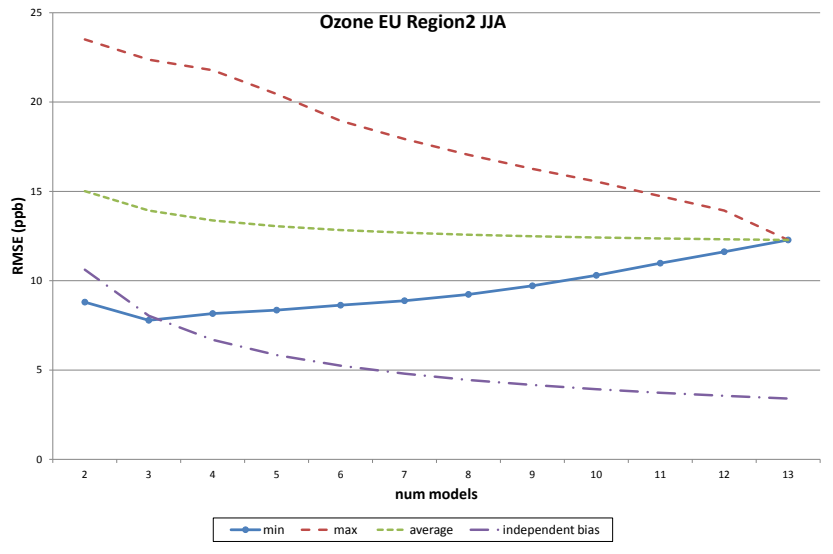

Fig. 8. Curves of maximum, mean and minimum RMSE for ozone in EU region 2. The curves are obtained by calculating the mean of randomly sampled subsets of models as a function of $m$, the dimension of the subsets. The theoretical decay that would occur if the model errors were independent, $\sim \mathrm{m}^{-0.5}$, is also reported.

can be moderated in case of applications to forecasting. In that case we could use a portion of the data to generate back $\bmod _{P C}$, and another portion for verification of the forecast. Current work is devoted to this aspect.

\subsection{Comparing the different methods: discussion}

\subsubsection{Member selection}

Members selected with MDS, HC, minRMSE and CAR score are reported in Table 4, where the redundancy index $\rho I$ of each reduced ensemble is also reported.

$\mathrm{HC}$ analysis of region 1 highlights that there is a group of two models common to the four pollutants (FI1 and FR3), and with the exception of $\mathrm{O}_{3}$, also DE1 and HR1 are in common. Furthermore, given the high level of similarity between DE2 and US4, $\mathrm{NO}_{2}$ and $\mathrm{SO}_{2}$ are represented by the same members (Table 4). The outputs of these models have therefore the least correlated bias, and in this sense can be considered non-redundant. For region 2 we found the selection to be more sensitive to the pollutant, with only the pair US3 and $\mathrm{DE} 1$ common to the species of $\mathrm{NO}_{2}$ and $\mathrm{SO}_{2}$. The spatial dependence of the bias is not entirely removed by the metric $d_{\mathrm{m}}$, as members of region 1 and 2 are quite different, with only three members in common for $\mathrm{O}_{3}$ (FI1, FR4, UK2) and CO (FI1, DE1, HR1). Members selected by HC and MDS are, in general, different.

The combination of minimum RMSE for region 1 is often achieved by combining the members that individually perform among the best. For example, for $\mathrm{NO}_{2}$ and $\mathrm{SO}_{2}$ the 
two models whose mean produce the minimum RMSE are ranked the highest for error. This is not the case for $\mathrm{O}_{3}$ and $\mathrm{CO}$. For the former, RMSE is minimised by the mean of the first three ranked (FR4, PL1, US3) with the last (DE1) and a middle ranked one (DK1), whilst for the latter the combination is composed by the best individual model (US4) with two middle ranked ones (FI1 and UK2). It is well established in information theory that good, larger feature sets do not necessarily include the good, small sets. Mathematically, the theorems by Elashoff et al. (1967), Cover (1976) and Toussaint (1971) had proven two important results on member selection and individual score of the members; that is, the best two models are not the two best and further that the best single model need not be in the best $k$. Therefore, the simple method of selecting just the best individual features may prove unsuccessful, although the selection of members based on performance might be justified in some cases (e.g. McSweeney et al., 2012). Pierce et al. (2009), in the context of climate modelling, showed that the mean of the best and that of the worst models that could be built out a large ensemble were statistically indistinguishable, and that the rank of the ensemble did not reflect that of the individual models. Similar conclusions were drawn by Solazzo et al. (2012a) for $\mathrm{O}_{3}$ in Europe and North America.

For some species, the minimum error is obtained by combining highly redundant members (Table 4), as, for example, $\mathrm{SO}_{2}$ and $\mathrm{NO}_{2}$ in region 2, where the two instances of WRF/Chem run by DE2 and US4 both participate to minimise the RMSE. As we can see from Fig. 4, these members (in the red square) are often those maximising the variance of the error. Because of the trade-off between bias, variance and covariance (Eq. 1), and due to the presence of negatively correlated members, the minimum RMSE is achieved by combining redundant and less redundant models. For example, DE3 errors are uncorrelated with the quintuplet of models minimising MSE for $\mathrm{SO}_{2}$ in region 2, while FR3 (which also belongs to the quintuplet) is highly redundant with respect to the others. Similar patterns are detected for the other compounds too. This is an additional indication that independence and skills need be investigated separately (Abramowitz, 2010). Only in the case of unbiased and positively correlated models is the error minimised by a null covariance term and thus by uncorrelated models.

Two similar, highly redundant models are bound to score identically under a variety of member selection techniques. This could be a possible way to read the combination of redundant members optimising the RMSE. We have applied the CAR score recently developed by Zuber and Strimmer (2011) to our dataset (available in the package "relaimpo" for the R statistical software, http://www.r-project.org). This method provides a ranking based on the partial correlation between model and the observations, conditioned to all other models. The CAR methodology is related to the amount of explained variance, enforces the simultaneous selection of highly correlated predictors and penalises variables correlat- ing with opposite signs with the observations. Models with a small CAR score contribute little to improve the prediction error or to reduce the unexplained variance. For the species $\mathrm{O}_{3}$ and $\mathrm{NO}_{2}$ of region 1 , and $\mathrm{O}_{3}, \mathrm{CO}$ and $\mathrm{NO}_{2}$ of region 2 , two models selected using the CAR score are in common with the selection based on minimum RMSE (the first four CAR-ranked models are reported in Table 4). Further to that, the overall redundancy of the ensemble built by the mean of the first four CAR-ranked models is, on some occasions, even lower than that of $\mathrm{HC}$ selection $\left(\mathrm{O}_{3}, \mathrm{SO}_{2}\right.$ and $\mathrm{NO}_{2}$ in region $1 ; \mathrm{CO}$ and $\mathrm{NO}_{2}$ in region 2).

\subsubsection{Skill scores}

In Table 5 we report the scores of the reduced ensemble generated with the methods discussed above. The full-member ensemble mean is also included as reference. With a few exceptions, the reduced ensembles score better than, or as well as, the full ensemble, especially in terms of variability. Overall, the minRMSE selection seems to outperform the other techniques for a number of pollutants in both regions. It gives the best accuracy (lowest error) by definition, and scores among the best also for variability. This is not surprising as accuracy implies precision (but the vice versa is not true). Good performance does not seem to be related to the redundancy of the ensembles as too low redundancy $\left(\mathrm{SO}_{2}\right.$, $\mathrm{CO}, \mathrm{NO}_{2}$ of region 1) does not systematically correspond to the best scores, enforcing the conclusion that diversity does not necessarily optimise skills such as accuracy due to negatively correlated members. This aspect deserves future investigations. HC, MDS and CAR methods do not consistently score high, although performing best on some occasions.

The overwhelming strength of the weighted PCA ensemble derives from having used the observations to rebuild the time series, as discussed in Sect. 5.4. In general, though, one of the main drawbacks of weighted ensembles is that they are not robust enough to be applied under a variety of scenarios (species, temporal, spatial), and in practical applications MM mean is often preferred (Pierce et al., 2009; Knutti et al., 2010).

\subsection{Implications for $A Q$ forecasting}

We outline here some considerations about applying the techniques of dimension reduction and member selection to periods of time other than those used for selecting the members. It is in the ensemble forecasting applications that the low redundancy of the bias plays the most important role: since observations are not available to provide evaluation, averaging out of errors is the only means to avoid common and redundant biases.

We thus ask whether any associativity among members can be inferred in the case where observational data were not available. In other words, knowing the associativity among the errors, what can be deducted about the associativity of 
Table 5. Ensemble skills for regions 1 and 2 of Europe (JJA). (RMSE: root-mean-square error; $R$ : Pearson correlation coefficient; NMB: normalised mean bias; STDEV ratio: modelled to observed standard deviation). Results in bold are those for which the selected ensemble scores better or as well as the full member ensemble (vice versa for the values in italic).

\begin{tabular}{|c|c|c|c|c|c|}
\hline EU region 1 & & RMSE & $R$ & NMB & STDEV ratio \\
\hline \multirow[t]{6}{*}{$\mathrm{CO}$} & PCA & 0.03 & 0.65 & 0.25 & 5.20 \\
\hline & $\mathrm{HC}$ & 0.06 & 0.36 & -0.22 & 0.39 \\
\hline & minRMSE & 0.05 & 0.36 & -0.09 & 0.45 \\
\hline & MDS & 0.06 & 0.28 & -0.19 & 0.51 \\
\hline & CAR & 0.06 & 0.41 & -0.29 & 0.44 \\
\hline & Full Ensemble & 0.06 & 0.38 & -0.26 & 0.39 \\
\hline \multirow{6}{*}{$\mathrm{O}_{3}$} & PCA & 2.5 & 0.99 & 0.04 & 0.99 \\
\hline & $\mathrm{HC}$ & 12.0 & 0.96 & 0.003 & 0.63 \\
\hline & $\operatorname{minRMSE}$ & 8.1 & 0.96 & 0.03 & 0.81 \\
\hline & MDS & 11.2 & 0.94 & 0.04 & 0.70 \\
\hline & CAR & 10.8 & 0.97 & -0.05 & 0.71 \\
\hline & Full Ensemble & 10.9 & 0.96 & 0.002 & 0.67 \\
\hline \multirow[t]{6}{*}{$\mathrm{SO}_{2}$} & PCA & 0.9 & 0.96 & 0.12 & 1.12 \\
\hline & $\mathrm{HC}$ & 2.0 & 0.17 & -0.07 & 0.57 \\
\hline & minRMSE & 1.9 & 0.27 & -0.11 & 0.55 \\
\hline & MDS & 2.1 & 0.16 & 0.12 & 0.60 \\
\hline & CAR & 2.2 & $<0.1$ & $-\mathbf{0 . 0 3}$ & 0.75 \\
\hline & Full Ensemble & 2.2 & 0.17 & 0.26 & 0.49 \\
\hline \multirow[t]{6}{*}{$\mathrm{NO}_{2}$} & PCA & 1.0 & 0.99 & 0.20 & 1.09 \\
\hline & $\mathrm{HC}$ & 3.5 & 0.68 & -0.09 & 1.05 \\
\hline & minRMSE & 3.0 & 0.74 & -0.09 & 0.96 \\
\hline & MDS & 4.7 & 0.61 & 0.20 & 1.43 \\
\hline & CAR & 3.6 & 0.74 & -0.24 & 0.95 \\
\hline & Full Ensemble & 3.7 & 0.67 & 0.18 & 1.06 \\
\hline EU region 2 & & RMSE & $R$ & NMB & STDEV ratio \\
\hline \multirow[t]{6}{*}{$\mathrm{CO}$} & PCA & 0.04 & 0.99 & 0.18 & 0.98 \\
\hline & $\mathrm{HC}$ & 0.05 & 0.48 & -0.21 & 0.68 \\
\hline & $\operatorname{minRMSE}$ & $\mathbf{0 . 0 3}$ & 0.38 & 0.02 & 0.83 \\
\hline & MDS & 0.04 & 0.45 & $-\mathbf{0 . 1 7}$ & 0.67 \\
\hline & CAR & 0.07 & 0.58 & -0.38 & 0.57 \\
\hline & Full Ensemble & 0.07 & 0.50 & -0.35 & 0.57 \\
\hline \multirow[t]{6}{*}{$\mathrm{O}_{3}$} & PCA & 2.5 & 0.98 & -0.005 & 0.99 \\
\hline & $\mathrm{HC}$ & 11.6 & 0.92 & 0.005 & 0.81 \\
\hline & minRMSE & 7.8 & 0.95 & 0.02 & 0.91 \\
\hline & MDS & 15.3 & 0.93 & -0.14 & 0.73 \\
\hline & CAR & 7.8 & 0.95 & 0.02 & 0.84 \\
\hline & Full Ensemble & 12.3 & 0.93 & -0.06 & 0.71 \\
\hline \multirow[t]{6}{*}{$\mathrm{SO}_{2}$} & PCA & 0.7 & 0.74 & 0.03 & 1.40 \\
\hline & $\mathrm{HC}$ & 0.7 & 0.73 & -0.13 & 3.30 \\
\hline & minRMSE & 0.5 & 0.59 & -0.07 & 1.08 \\
\hline & MDS & 0.8 & 0.53 & -0.3 & 0.86 \\
\hline & CAR & 0.8 & 0.76 & -0.4 & 1.11 \\
\hline & Full Ensemble & 0.8 & 0.59 & -0.4 & 0.77 \\
\hline \multirow[t]{6}{*}{$\mathrm{NO}_{2}$} & PCA & 1.0 & 0.99 & 0.5 & 1.03 \\
\hline & $\mathrm{HC}$ & 3.4 & 0.66 & 0.05 & 2.12 \\
\hline & minRMSE & 1.9 & 0.70 & -0.07 & 1.32 \\
\hline & MDS & 3.6 & 0.67 & 0.06 & 2.12 \\
\hline & CAR & 2.2 & 0.75 & -0.26 & 1.38 \\
\hline & Full Ensemble & 2.5 & 0.59 & -0.16 & 1.47 \\
\hline
\end{tabular}


the models underlining those errors? This problem is of direct relevance to forecasting, and thus worth investigating. The starting point is as usual the covariance matrix of the errors $\operatorname{cov}\left(d_{i}, d_{j}\right)$. After some basic manipulation we get

$\operatorname{cov}\left(d_{i}, d_{j}\right)=\operatorname{cov}\left(m_{i}, m_{j}\right)-\left(\operatorname{cov}\left(m_{i}, \mathrm{obs}\right)+\operatorname{cov}\left(m_{j}, \mathrm{obs}\right)\right)(12)$ $+\operatorname{var}(\mathrm{obs})=\operatorname{cov}\left(m_{i}, m_{j}\right)-\left(\operatorname{var}\left(d_{i}\right)+\operatorname{var}\left(d_{j}\right)\right)+\operatorname{var}(\mathrm{obs})$.

The model error covariance is strictly related to the the model covariance, thus we cannot prescind from the observations. All we can do is to infer some consideration about the covariance of the model errors for short periods of time ahead. In practical terms, we first derive a reduced ensemble from the matrix of errors $\operatorname{cov}\left(d_{i}, d_{j}\right)$. Then, if the trend of the error does not change drastically for a few hours or days ahead, we can deduce that the association among them does not change either, and thus the reduced ensemble is still the best option. Exploitation of reducing ensembles and member selection for forecasting applications is a topical argument and a matter of ongoing work.

Recently, Galmarini et al. (2013) have investigated the possibility of forecasting AQ starting from the combination of well-behaved spectral properties extracted from the AQMEII ensemble. The results show that the approach outruns even the ensemble median. Further investigation will be devoted to determining the correspondence between the reduced set obtained here and the properties of the ensemble put together by Galmarini et al. (2012b) for the sake of identifying a deeper structure inside in the model behaviour and performance.

\section{Conclusions}

The similarity of members in ensemble modelling is an outstanding issue which has recently raised awareness in the ensemble climate community but not in the AQ one. In this study we explain the risks of combining models sharing highly correlated bias into ensembles. We apply our analysis to a high-resolution dataset covering two regions of EU for 3 months. Along with observational data, we have treated results of $13 \mathrm{AQ}$ models for the air pollutants of $\mathrm{CO}, \mathrm{O}_{3}$, $\mathrm{NO}_{2}$ and $\mathrm{SO}_{2}$.

We have provided definitions for the concepts of independence, diversity/similarity, redundancy of models and their errors, which are often used interchangeably, giving raise to misconception. Due to practical difficulties in computing independency, we used the redundancy instead, which is simpler to handle and has the advantage of expressing the amount of the accounted-for variance. Conceptually we believe this is very important, as it allows for univocal interpretation of the results.

We started by applying the metric $d_{\mathrm{m}}$ introduced in climate modelling studies to our ensemble of regional-scale pollutant concentrations. $d_{\mathrm{m}}$ serves the scope of eliminating overarching commonalities among members and to explore hidden similarities, i.e. those underlying common modules and parameters in the models. Some main results and considerations are as follows:

1. The correlation among the majority of models remained a constant feature across the two examined regions, but varied from species to species. Generally it was not possible to identify model similarities common to the four species. This implies a large spectrum of partially shared modules and parameterisations within the AQ modelling systems which are invoked depending on the species and on other inputs, such as meteorology and emissions. Although most of the model similarities encapsulated by the multi-model ensemble mean error were removed by calculating $d_{\mathrm{m}}$, similarities among model errors were still found to be significant.

2. By projecting the observational values into the eigenvectors of the anomalies of the models about the MM ensemble, we found that the ensemble is wide; that is, it accounts for more variability than that of the observations. We concluded that the ensemble size, constrained by the available members, was too large. Given the massive application of AQ models in regulatory applications and the more and more stringent AQ targets, the detected overconfidence can have great cost. This, together with item 1 above, justify the need for the analysis of the redundancy of the datasets.

3. We explored some dimension-reduction methods:

- Eigenvalue methods - number of effective models and principal component analysis.

- Clustering analysis and dendrogram representation.

- Multi-dimensional scaling and graphical representation of model similarities as mutual distance among models.

- The heuristic minRMSE, determining the size of the ensemble of models whose mean minimise the RMSE.

None of the aforementioned method is new; they are all well-established techniques used, in many varieties, in various branches of science. They have neither been used before in an AQ ensemble context, nor compared. We also introduced, where possible, the nexus between these techniques and redundancy. We found that the optimal size of an ensemble of poorly correlated members is of about 4-6, implying that more than half of the information of the full MM ensemble is redundant.

4. We continued the investigation by applying memberselection techniques and scoring to the reduced ensembles against simple operational metrics, taking the 
scores of the full member ensemble mean as a benchmark. We proved that subsets of models outperform the full ensemble. The minRMSE selection seems to outperform the other techniques for a number of pollutants in both regions. It gives the best accuracy (lowest error) by definition, but scores among the best also for variability. HC, MDS and CAR methods do not consistently score high, although performing best in some occasions.

5. The error being minimised by highly redundant members does not justify, in our view, the use of the ensemble of those members. Skills and diversity need to be analysed in separation. This is because redundant members might share common biases which will force the agreement to be directed towards the same direction, with the risk of misjudging the results. These aspects are likely to be detected by a diagnostic type of analysis (rather that by simple operational scores based on distance metrics), and may often reveal more about the causes of model errors and the processes responsible for those errors (Dennis et al., 2010; Gleckler et al., 2008). The combination of minimum error being achieved by a highly redundant subset of models is due to the presence of negatively correlated members whose covariance minimise the trade-off between variance, covariance and bias. Further investigations need to be expanded to explain why highly redundant ensembles of negatively correlated models produce high accuracy.

6. Application of PCA to the matrix of errors for the purpose of data reduction has proved successful. By contrast, generating the reduced time series (the time series projected on the leading eigenvectors) is not trivial, and requires the use of the observational data, which masks the outcome of the procedure. As no applications of this sort have been found in the literature, our intention is to devote future work to this aspect which might be relevant in the realm of forecasting.

7. Finally, we have highlighted the steps for applying the methods of dimension reduction and member selection to a forecasting context.

We also believe the effort we spent to migrate some of the knowledge and techniques developed in other scientific areas (especially computer science, genetics and climate modelling) will contribute to raised awareness in the AQ community about the dependency of models and the meaning of model agreement.

Acknowledgements. The AQMEII community (http: //aqmeii.jrc.ec.europa.eu/) is kindly acknowledged for providing the data used in the analysis.

Edited by: J. Brandt

\section{References}

Abramowitz, G.: Model Independence in multi-model ensemble prediction, Australian Meteorological and Oceanographic Journal, 59, 3-6, 2010.

Annan, J. D. and Hargreaves, J. C.: Reliability of the CMIP3 ensemble, Geophys. Res. Lett., 37, L02703, doi:10.1029/2009GL041994, 2010.

Borg, I. and Groenen, P.: Modern Multidimensional Scaling: theory and applications (2nd ed), Springer-Verlag, New York, 2005.

Bretherton, C. S., Widmann, M., Dymnikov, V. P., Wallace, J. M., and Bladè, I.: The effective number of spatial degrees of freedom of a time-varying field, J. Climate, 12, 1990-2009, 1999.

Brown, G., Wyatt, J. L., and Tino, P.: Managing diversity in regression ensembles, Journal of Machine Learning Research, 6, 16211650, 2005.

Cattell, R. B.: The scree test for the number of factors, Multivariate Behavioural Research, 1, 245-276, 1966.

Côté, J., Gravel, S., Méthot, A., Patoine, A., Roch, M., and Staniforth, A.: The Operational CMC-MRB Global Environmental Multiscale (GEM) Model. Part I: Design Considerations and Formulation, Mon. Weather Rev., 126, 1373-1395, 1998.

Cover, T. T.: The best two independent measures are not the two best, IEEE Trans. System Man. and Cybernetics, 4, 116-117, 1974.

Cover, T. and Thomas, J.: Elements of Information Theory, 2nd ed., Wiley-Interscience, Hoboken, NJ, 2006.

Dennis, R., Fox, T., Fuentes, M., Gilliland, A., Hanna, S., Hogrefe, C., Irwin, J., Rao, S. T., Scheffe, R., Schere, K., Steyn, D., and Venkatram, A.: A framework for evaluating regional-scale numerical photochemical modelling systems, Environ. Fluid Mech., 10, 471-489, doi:10.1007/s10652-009-9163-2, 2010.

Ding, C. and He, X.: K-means clustering via Principal component analysis, Proceedings of the 21st International Conference on Machine Learning, Banff, Canada, 2004.

Ding, C. and Peng, H.: Minimum Redundancy feature selection from microarray gene expression data, Journal of Bioinformatics and Computational Biology, 3, 185-205, 2005.

Elashoff, J. D., Elashoff, R. M., and Goldman, G. E.: On the choice of variables in classification problems with dichotomous variables, Biometrika, 54, 668-670, 1967.

Fern, X. Z. and Brodley, C. E.: Solving cluster ensemble problems by bipartite graph partitioning, in Proceedings of 21th International Conference on Machine Learning (ICML2004), 2004.

Fiore, A. M., Dentener, F. J., wild, O., Cuvelier, C., Schultz, M. G., Hess, P., Textor, C., Schulz, M., Doherty, R. M., Horowitz, L. W., MacKenzie, I. A., Sanderson, M. G., Shindell, D. T., Stevenson, D. S., Szopa, S., Van Dingenen, R., Zeng, G., Atherton, C., Bergmann, D., Bey, I., Carmichael, G., Collins, W. J., Duncan, B. N., Faluvegi, G., Folberth, G., Gauss, M., Gong, S., Hauglustaine, D., Holloway, T., Isaksen, I. S. A., Jacob, D. J., Jonson, J. E., Kaminski, J. W., Keating, T. J., Lupu, A., Marmer, E., Montanaro, V., Park, R. J., Pitari, G., Pringle, K. J., Pyle, J. A., Schroeder, S., Vivanco, M. G., Wind, P., Wojcik, G., Wu, S., and Zuber, A.: Multimodel estimates of intercontinental sourcereceptor relationships for ozone pollution, J. Geophys. Res., 114, D04301, doi:10.1029/2008JD010816, 2009.

Galmarini, S., Bianconi, R., Appel, W., Solazzo, E., Mosca, S., Grossi, P., Moran, M., Schere, K., and Rao, S. T.: ENSEMBLE and AMET: two systems and approaches to a harmonised, sim- 
plified and efficient assistance to air quality model developments and evaluation, Atmos. Environ., 53, 51-59, 2012.

Galmarini, S., Kioutsioukis, I., and Solazzo, E.: E pluribus unum*: ensemble air quality predictions, Atmos. Chem. Phys., 13, 71537182, doi:10.5194/acp-13-7153-2013, 2013.

Garaud, D. and Mallet, V.. Automatic calibration of an ensemble for uncertainty estimation and probabilistic forecast: Application to air quality, J. Geophys. Res., 116, D19304, doi:10.1029/2011JD015780, 2011.

Gleckler, P. J., Taylor, K. E., and Doutriaux, C.: Performance metrics for climate models, J. Geophys. Res., 113, D06104, doi:10.1029/2007JD008972, 2008.

Groenen, P. J. F and van de Velden, M.: Multidimensional Scaling. Erasmus University Rotterdam, Econometric Institute, Econometric Institute Report EI 2004-15, 2004.

Grömping, U.: Estimator of relative importance in linear regression based on variance decomposition, The American Statistician, 61, 139-147, 2007.

Guenther, A., Zimmerman, P., and Wildermuth, M.: Natural volatile organic compound emission rate estimates for US woodland landscapes, Atmos. Environ., 28, 1197-1210, 1994.

Guttman, L.: Some necessary conditions for common-factor analysis, Psychometrika, 19, 149-161, 1954.

Jolliffe, I.: Principal component analysis, Springer, 2nd edition, 2002.

Jong, J.-C. and Kotz, S.: On a relation between principal components and regression analysis, The American Statistician, 53, 349-351, 1999.

Jun, M., Knutti, R., and Nychka, D. W.: Local eigenvalue analysis of CMIP3 climate model errors, Tellus, 60, 992-1000, 2008.

Kaiser, H.: The application of electronic computers to factor analysis, Educational and Psychological Measurement, 20, 141, 1960.

Kaminski, J. W., Neary, L., Struzewska, J., McConnell, J. C., Lupu, A., Jarosz, J., Toyota, K., Gong, S. L., Côté, J., Liu, X., Chance, K., and Richter, A.: GEM-AQ, an on-line global multiscale chemical weather modelling system: model description and evaluation of gas phase chemistry processes, Atmos. Chem. Phys., 8, 3255-3281, doi:10.5194/acp-8-3255-2008, 2008.

Knutti, R.: The end of model democracy?, Climate Change, 102, 395-404, 2010.

Knutti, R., Furrer, R., Tebaldi, C., Cermak, J., and Meehl, G.: Challenges in Combining Projections from Multiple Climate Models, J. Climate, 23, 2739-2758, 2010.

Kong, W., Vanderburg, C. R., Gunshin, H., Rogers, J. T., and Huang, X.: A Review of independent component analysis application to microarray gene expression data, BioTechniques, 45, 501-520, 2008.

Legendre, P. and Legendre, L. F. J.: Numerical Ecology, Elsevier Science BV, Amsterdam, Chapter 11, 853 pp., 1998.

Liu, Y. and Yao, X.: Ensemble learning via negative correlation, Neural Networks, 12, 1399-1404, 1999.

McSweeney, C. F., Jones, R. G., and Booth, B. B. B.: Selecting ensemble members to provide regional climate change information, J. Climate, 25, 7100-7121, 2012.

Moesa, H. A., Dukka Bahadur, K. C., and Akutsu, T.: Efficient determination of cluster boundaries for analysis of gene expression profile data using hierarchical clustering and wavelet transformation, Genome Informatics, 16, 132-141, 2005.
Murtagh, F.: Complexities of Hierarchic Clustering Algorithms: the state of the art, Computational Statistics Quarterly, 1, 101-113, 1984.

Nenes, A., Pilinis, C., and Pandis, S.: ISORROPIA: a new thermodynamic equilibrium model for multicomponent inorganic aerosols, Aquat. Geochem., 4, 123-152, 1998.

Peng, H., Long, F., and Ding, C.: Feature selectionbased on mutual information: criteria of Max-dependency, Max-relevance, and Min-redundancy, IEEE Transactions on Pattern Analysis and Machine Intelligence, 27, 1226-1238, 2005.

Pennel, C. and Reichler, T.: On the effective numbers of climate models, J. Climate, 24, 2358-2367, 2011.

Pierce, D. W., Barnett, T. P., Santer, B. D., and Gleckler, P. J.: Selecting global climate models for regional climate change studies, $\mathrm{P}$. Natl. Acad. Sci. USA, 106, 8441-8446, 2009.

Pirtle, Z., Meyer, R., and Hamilton, A.: What does it mean when climate models agree? A case for assessing independence among general circulation models, Environmental Science and Policy, 799, 351-361, 2010.

Potempski, S. and Galmarini, S.: Est modus in rebus: analytical properties of multi-model ensembles, Atmos. Chem. Phys., 9, 9471-9489, doi:10.5194/acp-9-9471-2009, 2009.

Potempski, S., Galmarini, S., Addis, R., Astrup, P., Bader, S., Bellasio, R., Bianconi, R., Bonnardot, F., Buckley, R., D'Amours, R., van Dijk, A., Geertsema, G., Jones, A., Kaufmann, P., Pechinger, U., Persson, C., Polreich, C., Prodanova, M., Robertson, L., Sørensen, J., Syrakov, D.: Multi-model ensemble analysis of the ETEX-2 experiment, Atmos. Environ., 42, 7250-7265, 2008.

Rao, S. T., Galmarini, S., and Puckett, S.: Air quality model evaluation international initiative (AQMEII), B. Am. Meteorol. Soc., 92, 23-30, 2011.

Riccio, A., Ciaramella, A., Giunta, G., Galmarini, S., Solazzo, E., and Potempski, S.: On the systematic reduction of data complexity in multi-model ensemble atmospheric dispersion modelling, J. Geophys. Res., 117, D05314, doi:10.1029/2011JD016503, 2012.

Simpson, D., Guenther, A., Hewitt, C. N., and Steinbrecher, R.: Biogenic emissions in Europe. 1. Estimates and uncertainties, J. Geophys. Res., 100D, 22875-22890, 1995.

Solazzo, E., Bianconi, R., Vautard, R., Appel, K. W., Moran, M. D., Hogrefe, C., Bessagnet, B., 5 Brandt, J., Christensen, J. H., Chemel, C., Coll, I., van der Gon, H. D., Ferreira, J., Forkel, R., Francis, X. V., Grell, G., Grossi, P., Hansen, A. B., Jericevic, A., Kraljevic, L., Miranda, A. I., Nopmongcol, U., Pirovano, G., Prank, M., Riccio, A., Sartelet, K. N., Schaap, M., Silver, J. D., Sokhi, R. S., Vira, J., Werhahn, J., Wolke, R., Yarwood, G., Zhang, J., Rao, S. T., and Galmarini, S: Ensemble modelling of surface level ozone in Europe and North America in the context of AQMEI, Atmos. Environ., 53, 60-74, 2012a.

Solazzo, E., Bianconi, R., Pirovano, G., Matthias, V., Vautard, R., Moran, M. D., Appel, K. W., Bessagnet, B., Brandt, J., Christensen, J. H., Chemel, C., Coll, I., Ferreira, J., Forkel, R., Francis, X. V., Grell, G., Grossi, P., Hansen, A. B., Hogrefe, C., Miranda, A. I., Nopmongco, U., Prank, M., Sartelet, K. N., Schaap, M., Silver, J. D., Sokhi, R. S., Vira, J., Werhahn, J., Wolke, R., Yarwood, G., Zhang, J., Rao, S. T., and Galmarini, S: Operational Model evaluation for particulate matter In europe and North America in the context of AQMEII, Atmos. Environ., 53, 75-92, $2012 \mathrm{~b}$. 
Solazzo, E., Bianconi, R., Pirovano, G., Moran, M. D., Vautard, R., Hogrefe, C., Appel, K. W., Matthias, V., Grossi, P., Bessagnet, B., Brandt, J., Chemel, C., Christensen, J. H., Forkel, R., Francis, X. V., Hansen, A. B., McKeen, S., Nopmongcol, U., Prank, M., Sartelet, K. N., Segers, A., Silver, J. D., Yarwood, G., Werhahn, J., Zhang, J., Rao, S. T., and Galmarini, S.: Evaluating the capability of regional-scale air quality models to capture the vertical distribution of pollutants, Geosci. Model Dev., 6, 791-818, doi:10.5194/gmd-6-791-2013, 2013.

Stewart, D. K. and Love, W. A.: A General Canonical Correlation Index, Psychol. Bullet., 70, 160-163, 1968.

Talagrand, O., Vautard, R., and Strauss, B.: Evaluation of probabilistic prediction systems, paper presented at aa seminar on predictability, Eur. cent. For Medium Weather Forecasting, Reading (UK), 1998.

Tebaldi, C. and Knutti, R.: The use of multi-model ensemble in probabilistic climate projections, Philos. Tr. Roy. Soc., 365A, 2053-2075, 2007.

Tinsley, H. E. A. and Brown, S. D.: Handbook of applied multivariate statistics and mathematical modeling, Academic Press, California (USA), 334, 338, 2000.

Toussaint, G. T.: Note on optimal selection of independent binary valued features for pattern recognition. IEEE Transactions on Information Theory, Vol. IT-17, 618, 1971.

Van Loon, M., Vautard, R., Schaap, M., Bergstrom, R., Bessagnet, B., Brandt, J., Builtjes, P. J., H., Christensen, J. H., Cuvelier, C., Graff, A., Jonson, J. E., Krol, M., Langner, J., Roberts, P., Rouil, L., Stern, R., Tarrason, L., Thunis, P., Vignati, E., White, L., and Wind, P.: Evaluation of long-term ozone simulations from seven regional air quality models and their ensemble average, Atmos. Environ., 41, 2083-2097, 2007.
Vautard, R., Schaap, M., Bergström, R., Bessagnet, B., Brandt, J., Builtjes, P. J. H., Christensen, J. H., Cuvelier, C., Foltescu, V., Graf, A., Kerschbaumer, A., Krol, M., Roberts, P., Rouïl, L., Stern, R., Tarrason, L., Thunis, P., Vignati, E., and Wind, P.: Skill and uncertainty of a regional air quality model ensemble, Atmos. Environ., 43, 4822-4832, 2009.

Vautard, R., Moran, M. D., Solazzo, E., Gilliam, R. C., Matthias, V., Bianconi, R., Chemel, C., Ferreira, J., Geyer, B., Hansen, A. B., Jericevic, A., Prank, M., Segers, A., Silver, J. D., Werhahn, J., Wolke, R., Rao, S. T., and Galmarini, S.: Evaluation of the meteorological forcing used for AQMEII air quality simulations, Atmos. Environ., 53, 15-37, 2012.

Yoon, S. and Kim, S.: Mutual information-based SVM-RFE for diagnostic classification of digitized mammograms, Pattern Recognition Letters, 30, 1489-1495, 2009.

Youness, G. and Saporta, G.: Comparing partitions of two sets of units based on the same variables, Adv Data Anal Classif., doi:I0.1007/s11634-009-0057-4, 2010.

Zhang, L., Gong, S., Padro, J., and Barrie, L.: A size-segregated particle dry deposition scheme for an atmospheric aerosol module, Atmos. Environ., 549-560, 2001.

Zuber, V. and Strimmer, K.: High-Dimensional Regression and variable selection using CAR scores, Statistical Applications in Genetics and Molecular Biology, 10, 1-25, 2011. 